\title{
SUDARE-VOICE variability-selection of active galaxies in the Chandra Deep Field South and the SERVS/SWIRE region
}

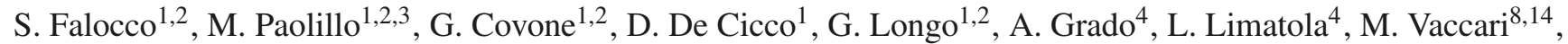 \\ M. T. Botticella ${ }^{4}$, G. Pignata ${ }^{5,6}$, E. Cappellaro ${ }^{7}$, D. Trevese ${ }^{9}$, F. Vagnetti ${ }^{10}$, M. Salvato ${ }^{11}$, M. Radovich ${ }^{7}$, L. Hsu $^{11}$, \\ M. Capaccioli ${ }^{1,2,4}$, N. Napolitano ${ }^{4}$, W. N. Brandt ${ }^{12,13}$, A. Baruffolo ${ }^{7}$, E. Cascone ${ }^{4}$, and P. Schipani ${ }^{4}$
}

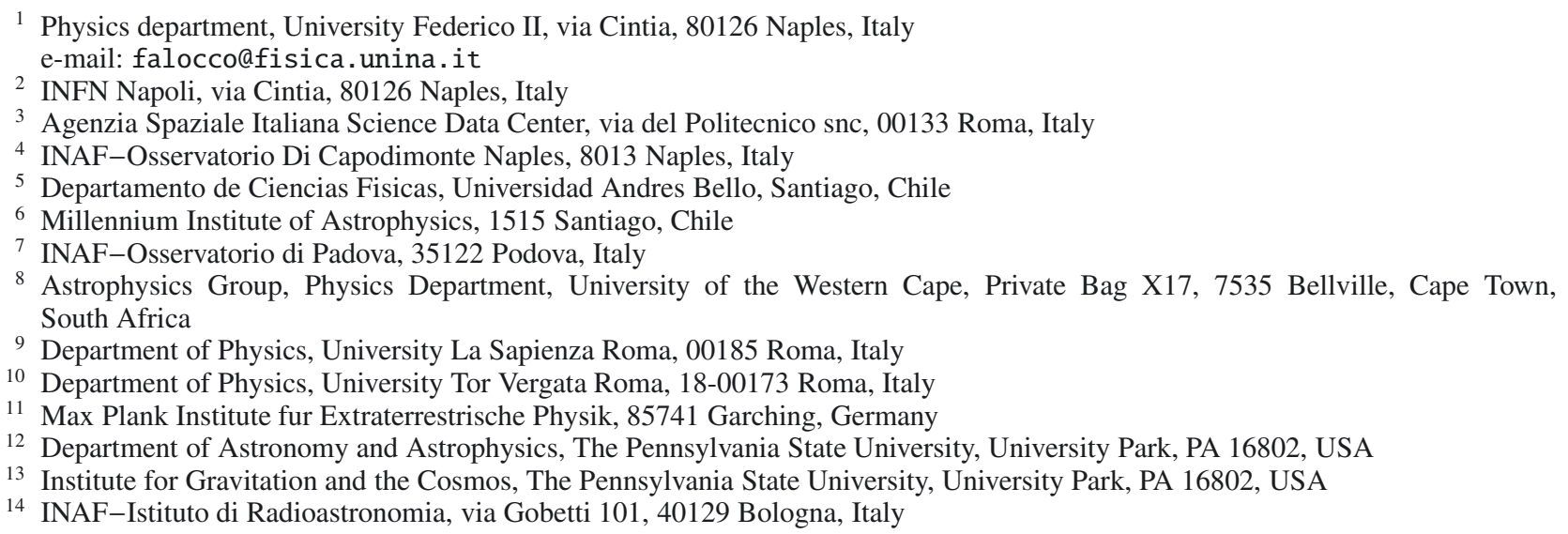

Received 5 October 2014 / Accepted 11 May 2015

\begin{abstract}
Context. One of the most peculiar characteristics of active galactic nuclei (AGNs) is their variability over all wavelengths. This property has been used in the past to select AGN samples and is foreseen to be one of the detection techniques applied in future multi-epoch surveys, complementing photometric and spectroscopic methods.

Aims. In this paper, we aim to construct and characterise an AGN sample using a multi-epoch dataset in the $r$ band from the SUDAREVOICE survey.

Methods. Our work makes use of the VST monitoring programme of an area surrounding the Chandra Deep Field South to select variable sources. We use data spanning a six-month period over an area of 2 square degrees, to identify AGN based on their photometric variability.

Results. The selected sample includes 175 AGN candidates with magnitude $r<23$ mag. We distinguish different classes of variable sources through their lightcurves, as well as X-ray, spectroscopic, SED, optical, and IR information overlapping with our survey. Conclusions. We find that $12 \%$ of the sample (21/175) is represented by supernovae (SN). Of the remaining sources, $4 \%(6 / 154)$ are stars, while 66\% (102/154) are likely AGNs based on the available diagnostics. We estimate an upper limit to the contamination of the variability selected AGN sample $\simeq 34 \%$, but we point out that restricting the analysis to the sources with available multi-wavelength ancillary information, the purity of our sample is close to $80 \%$ (102 AGN out of 128 non-SN sources with multi-wavelength diagnostics). Our work thus confirms the efficiency of the variability selection method, in agreement with our previous work on the COSMOS field. In addition we show that the variability approach is roughly consistent with the infrared selection.
\end{abstract}

Key words. galaxies: active - surveys - infrared: galaxies

\section{Introduction}

Active galactic nuclei (AGNs) are the most luminous persistent $^{1}$ sources in the Universe, and their emission is considered to be produced through accretion onto a super massive black hole (SMBH; Shakura \& Sunyaev 1976). Traditionally AGNs have been selected and classified on the basis of their optical emission lines (e.g. Veilleux \& Osterbrock 1987). However, the discovery and early investigation of these sources was based on broad-band photometry and the characteristic UV excess that

\footnotetext{
1 Fast transients, such as supernovae ( $\mathrm{SNe}$ ) or gamma ray bursts (GRB) can emit more energy on short timescales.
}

originates in the accretion disk (Markarian 1967). Since then, optical colours have been broadly used to select AGNs and are expected to play a major role in future astronomical surveys, such as those foreseen for LSST (Brandt et al. 2002). As a larger portion of the electromagnetic spectrum became accessible to astronomical observations, additional selection methods have been devised.

$\mathrm{X}$-ray emission, at least at bright luminosities and moderate gas column densities $\left(L_{\mathrm{X}}>10^{42} \mathrm{erg} \mathrm{s}^{-1}\right.$ and $n_{\mathrm{H}}<$ $10^{23} \mathrm{~cm}^{-2}$ ), is a most direct evidence of the presence of ongoing mass accretion. At low X-ray luminosities, starburst galaxies may contaminate the samples of AGN selected in soft X-rays 
(0.5-2. keV) (see e.g. Cerviño et al. 2002; Jiménez-Bailón et al. 2005; Lehmer et al. 2012) but such contamination is reduced above $2 \mathrm{keV}$ (Perez-Olea \& Colina 1996). For this reason, hard $\mathrm{X}$-ray surveys represent an effective method to provide a census of AGN, especially at high redshift where soft X-rays are shifted outside the observing band. For instance, deep observations of the Chandra Deep Field South (CDFS) area have been made by Chandra (Xue et al. 2011; Giacconi et al. 2002; Luo et al. 2008) and XMM-Newton (Comastri et al. 2011), allowing AGNs to be identified down to $L_{X}=10^{41} \mathrm{erg} \mathrm{s}^{-1}$ up to redshift $\sim 1$. The Swift Burst Alert Telescope has provided an all-sky survey in the hard X-rays (above $2 \mathrm{keV}$ ) allowing absorbed AGN (especially for $z<0.2$ ) and AGN to be sampled with X-ray luminosities $L_{\mathrm{X}}$, spanning values from $10^{42} \mathrm{erg} / \mathrm{s}$ to $10^{44} \mathrm{erg} / \mathrm{s}$ for $z<0.02$ (Ajello et al. 2012).

To overcome the biases introduced by dust and gas obscuration, which will affect UV, optical, and X-ray selection methods in different ways, infrared (IR) observations are commonly used, since the dusty torus that makes AGN difficult to select at ultraviolet and optical wavelengths emits radiation between 1.5 and $100 \mu \mathrm{m}$ (Sanders et al. 1989). For instance, Lacy et al. (2004) used mid-IR fluxes to construct diagnostics in order to separate AGN and galaxies in the Spitzer Space Telescope First Look Survey, relying on the different temperatures of dust in the circumnuclear and star-forming regions. The use of IR colour-selection criteria was refined in Stern et al. (2005), which reached an $80 \%$ completeness (of the spectroscopically identified unabsorbed AGN sample) with less than $20 \%$ contamination. By studying the large multi-wavelength dataset in the Chandra/SWIRE Survey $\left(0.6 \mathrm{deg}^{2}\right.$ in the Lockman Hole), Polletta et al. (2006) found a population of highly absorbed (Compton-thick) AGNs with this method. On the other hand, they found that the completeness of their IR-selected sample is $40 \%$ with respect to the X-ray selected heavily absorbed AGN in that field, indicating that a large number of these sources are elusive even in the mid-IR.

An alternative approach for searching AGN is based on the fact that the luminosity of most AGN intrinsically vary at all wavelengths (see e.g. Kawaguchi et al. 1998; Paolillo et al. 2004; García-González et al. 2014; Ulrich et al. 1997, and references therein), thus making variability one of the most distinctive properties of these sources. It is known for causality arguments that the fluctuations observed on timescales of days and months come from relatively small regions, such as the accretion disk and/or the torus. Although the physical interpretation of AGN variability is still poorly understood, in radio-quiet AGN, the accretion rate and instabilities in the accretion flow seem to play a fundamental role (see, e.g. Peterson 2001, and references therein); in jet-dominated sources, on the other hand, variability can be produced by relativistic effects (see e.g. Ulrich et al. 1997; Gopal-Krishna \& Subramanian 1991; Qian et al. 1991; Peterson 2001).

The variability selection method is thus based on the assumption that all AGN vary intrinsically in the observed band, without requiring assumptions on the spectral shape, colours, and/or line ratios. On the other hand, the method is biased against absorbed AGNs (e.g. Type II) since their primary emission from the nucleus is absorbed along the line of sight, thus any optical variability from these sources is very hard to detect. Variability selection helps in selecting those AGNs that can be confused with stars or galaxies of similar colours: the combination of variability selection technique with the multi-band photometry allows separating AGN from this class of contaminants (Ivezić et al. 2003; Young et al. 2012; Antonucci et al. 2015).
Several authors (Hawkins 1983; Trevese et al. 1989; Veron \& Hawkins 1995; Bershady et al. 1998; Geha et al. 2003; Sesar et al. 2007; Graham et al. 2014) have used variability-selection techniques to verify the completeness of colour-colour and spectroscopic selection techniques for high luminosity AGNs, for which the luminosities of the nuclei are well above those of the host galaxies. Other works (Sarajedini et al. 2003, 2006, 2011; Villforth et al. 2012) extend such analysis to low-luminosity AGNs. Variability selection methods have revealed to be useful to complete the demography of low-luminosity AGNs. This is in part because variability helps to detect AGN against the host galaxy contamination and also because, as shown by Trevese et al. (1994), for example, variability is intrinsically stronger in lower luminosity sources.

In this paper we aim at constructing a new variabilityselected AGN sample exploiting the data from the ongoing SUDARE-VOICE survey performed with the VLT Survey Telescope (VST). SUDARE (Botticella et al. 2013 and Cappellaro et al., in prep.) is a VST monitoring survey aimed at searching for supernovae ( $\mathrm{SNe}$ ) at intermediate redshift, through observations in the $g, r$, and $i$ bands in the region of the sky surrounding the CDFS (see Fig. 1). The VOICE survey (Vaccari et al., in prep.) is an independent effort aimed at providing UV-optical $(u, g, r)$ coverage of two selected areas in the southern hemisphere: the extended CDFS (ECDFS) and the ELAIS South 1. The two surveys have joined efforts on the ECDFS to optimise the use of VST telescope time, providing both timeresolved and deep optical-UV observations over several square degrees to achieve different scientific goals.

A companion paper by De Cicco et al. (2015, hereafter Paper I), studied the variability-selected sources in the COSMOS region $\left(1 \times 1 \mathrm{deg}^{2}\right)$ monitored by the SUDARE survey. In Paper I, the sample of variable sources was validated mainly through a comparison with X-ray-selected samples. The present paper focuses on the part of the SUDARE-VOICE monitoring programme covering $2 \mathrm{deg}^{2}$ around the CDFS. The region studied in the present paper has limited X-ray coverage, but has a significant overlap with the IR and optical surveys SWIRE (Lonsdale et al. 2004) and SERVS (Mauduit et al. 2012), which provide excellent optical/IR ancillary data. Since the area studied here is larger than the COSMOS region, this survey is best suited to strengthening the results of Paper I and to extending the comparison of variability selection with other optical and IR selection techniques.

The paper is structured as follows. In Sect. 2 we describe the survey, in Sect. 3 the methodology used for the data analysis, in Sect. 4 the selection of variable sources, and in Sect. 5 the validation of the catalogue, and finally our conclusions and the summary are given in Sect. 6. In the following we use the AB system, unless stated otherwise.

\section{Data}

This work is based on data in the $r$ band from the SUDAREVOICE survey performed with the VST telescope (Botticella et al. 2013; Cappellaro et al., in prep.; Vaccari et al., in prep.). VST telescope is equipped with the OmegaCAM detector (Kuijken 2011), composed of 32 CCDs with a total field-of-view (FOV) of $1^{\circ} \times 1^{\circ}$. The data are centred on the Chandra Deep Field South, covering an area of $2 \mathrm{deg}^{2}$, in the $u, g, r, i$ bands. Table 1 summarises the observations in the $r$ band used in this work. The observations are spread over two adjacent fields (labelled here CDFS1 and CDFS2), and as the table shows, they span five months in the CDFS1 and four for CDFS2. The observations at 
Table 1. Observations in the $r$ band used in the present work.

\begin{tabular}{|c|c|c|c|c|}
\hline $\begin{array}{l}\text { OB } \\
\text { (1) }\end{array}$ & $\begin{array}{c}\text { Date } \\
(2) \\
y-m-d \\
\end{array}$ & $\begin{array}{l}\text { Field } \\
\text { (3) }\end{array}$ & $\begin{array}{c}T_{\exp } \\
(4) \\
\mathrm{S} \\
\end{array}$ & $\begin{array}{c}F W H M \\
(5) \\
\operatorname{arcsec} \\
\end{array}$ \\
\hline 703642 & 2012-08-10 & CDFS1 & 1800.0 & 0.806 \\
\hline 703646 & $2012-08-13$ & CDFS1 & 1800.0 & 0.693 \\
\hline 703664 & 2012-09-02 & CDFS1 & 1800.0 & 1.019 \\
\hline 703674 & 2012-09-08 & CDFS1 & 1800.0 & 0.998 \\
\hline 703682 & 2012-09-14 & CDFS1 & 1800.0 & 0.550 \\
\hline 703686 & $2012-09-17$ & CDFS1 & 1800.0 & 1.062 \\
\hline 703690 & 2012-09-20 & CDFS1 & 1800.0 & 0.874 \\
\hline 703694 & 2012-09-22 & CDFS1 & 1800.0 & 0.892 \\
\hline 779894 & 2012-10-07 & CDFS1 & 1800.0 & 0.927 \\
\hline 779919 & 2012-10-08 & CDFS1 & 1800.0 & 0.930 \\
\hline 779898 & $2012-10-11$ & CDFS1 & 1800.0 & 0.921 \\
\hline 779902 & $2012-10-15$ & CDFS1 & 1800.0 & 1.034 \\
\hline 779906 & $2012-10-17$ & CDFS1 & 1800.0 & 0.924 \\
\hline 779910 & $2012-10-21$ & CDFS1 & 1800.0 & 0.508 \\
\hline 779914 & $2012-10-25$ & CDFS1 & 1800.0 & 0.862 \\
\hline 843920 & 2012-11-04 & CDFS1 & 1800.0 & 0.671 \\
\hline 843925 & 2012-11-06 & CDFS1 & 1800.0 & 0.832 \\
\hline 843930 & 2012-11-08 & CDFS1 & 1800.0 & 0.884 \\
\hline 843935 & 2012-11-10 & CDFS1 & 1800.0 & 0.762 \\
\hline 843945 & 2012-11-19 & CDFS1 & 1800.0 & 0.658 \\
\hline 779831 & $2012-12-03$ & CDFS1 & 1800.0 & 0.708 \\
\hline 779836 & $2012-12-07$ & CDFS1 & 1800.0 & 0.812 \\
\hline 779846 & $2012-12-13$ & CDFS1 & 1800.0 & 0.545 \\
\hline 779856 & $2012-12-20$ & CDFS1 & 1800.0 & 0.963 \\
\hline 779862 & 2013-01-03 & CDFS1 & 1800.0 & 0.684 \\
\hline 779867 & 2013-01-06 & CDFS1 & 1800.0 & 0.913 \\
\hline 779872 & 2013-01-10 & CDFS1 & 1800.0 & 0.888 \\
\hline stacked & & CDFS1 & 18360.0 & 0.671 \\
\hline 606786 & 2011-10-20 & CDFS2 & 1800.0 & 1.173 \\
\hline 606792 & $2011-10-25$ & CDFS2 & 1800.0 & 0.560 \\
\hline 606795 & $2011-10-28$ & CDFS2 & 1800.0 & 0.918 \\
\hline 606798 & 2011-10-30 & CDFS2 & 1800.0 & 1.064 \\
\hline 606801 & 2011-11-02 & CDFS2 & 1800.0 & 0.779 \\
\hline 606804 & 2011-11-04 & CDFS2 & 1800.0 & 0.616 \\
\hline 606808 & 2011-11-15 & CDFS2 & 1800.0 & 0.607 \\
\hline 606811 & 2011-11-18 & CDFS2 & 1800.0 & 0.897 \\
\hline 606814 & 2011-11-21 & CDFS2 & 1800.0 & 0.680 \\
\hline 606817 & 2011-11-23 & CDFS2 & 1800.0 & 0.903 \\
\hline 606820 & 2011-11-26 & CDFS2 & 1800.0 & 0.638 \\
\hline 606823 & $2011-11-28$ & CDFS2 & 1800.0 & 1.043 \\
\hline 606826 & 2011-12-01 & CDFS2 & 1800.0 & 0.824 \\
\hline 606829 & $2011-12-03$ & CDFS2 & 1800.0 & 0.523 \\
\hline 606723 & 2011-12-14 & CDFS2 & 2160.0 & 0.883 \\
\hline 606727 & 2011-12-17 & CDFS2 & 1800.0 & 0.880 \\
\hline 606756 & 2012-01-14 & CDFS2 & 1800.0 & 0.769 \\
\hline 606760 & 2012-01-18 & CDFS2 & 1800.0 & 0.574 \\
\hline 606764 & $2012-01-20$ & CDFS2 & 1800.0 & 1.003 \\
\hline 606768 & $2012-01-23$ & CDFS2 & 1800.0 & 0.594 \\
\hline 606772 & $2012-01-25$ & CDFS2 & 1800.0 & 0.901 \\
\hline 606776 & $2012-01-29$ & CDFS2 & 1800.0 & 0.666 \\
\hline stacked & & CDFS2 & 19440.0 & 0.637 \\
\hline
\end{tabular}

Notes. Columns: (1): observing block i.d. (OB) of each epoch; (2): date of observation of each epoch; (3): target field; (4): total exposure time; (5): median seeing of the epoch.

each epoch are composed of five dithers with an exposure time of $30 \mathrm{~min}$ in total. Hereafter, we refer to each epoch with the corresponding VST observing block number (OB). The observations in the $r$ band were performed approximately every three days, avoiding the ten days around the full moon. Observations

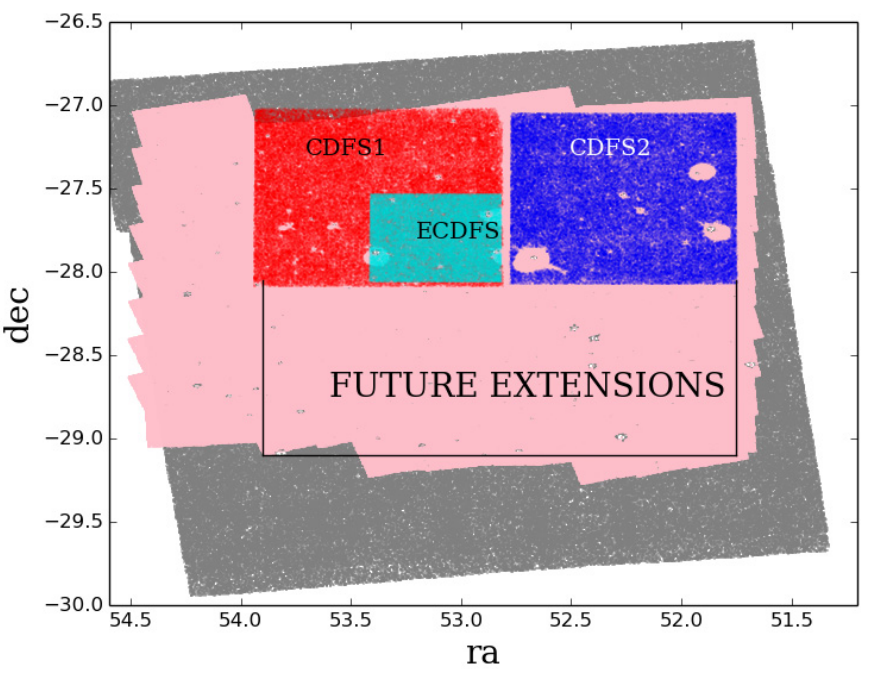

Fig. 1. Region covered by the VST observations compared to other overlapping surveys. Red: VST-CDFS1, blue: VST-CDFS2, grey: SWIRE, pink: SERVS, cyan: ECDFS area. The holes in the CDFS1 and CDFS2 are due to the masks we applied to exclude bright stars, spikes, and reflection features, as explained in detail in Sect. 3.

in the $g$ and $i$ filters were taken every ten days; instead, the $u$ band observations from VOICE do not have a specific cadence. For our variability analysis, we chose to use the $r$ band data, due to the better temporal sampling.

As described in detail in De Cicco et al. (2015), the data reduction was performed by using VST-Tube, a pipeline specifically designed to reduce VST/Omegacam data (Grado et al. 2012), which takes care of the instrumental signature removal, including overscan, bias and flat- field correction, CCD gain harmonisation and illumination correction (IC), co-addition of the exposures for each epoch, and finally astrometric and photometric calibration of the data.

We note that in the case of wide field images, the final photometric accuracy across the entire FOV depends on the quality of the gain harmonization across the multiple CCDs, as well as on the illumination and scattered light correction, which are position and time dependent. The absolute photometric calibration was computed on the photometric night 2011 Dec. 17 by comparing the observed magnitude of stars in photometric standard fields with SDSS DR8 magnitudes (Eisenstein et al. 2011). The extinction coefficient was taken from the extinction curve M.OMEGACAM.2011-12-01T16:15:04.474 provided by ESO. The relative photometric correction among the exposures was obtained by minimising the quadratic sum of differences in magnitude of the multiple detections. The dependence of such corrections on the position within the FOV is taken care of during the gain harmonisation and IC correction phase mentioned earlier. We had a total of 29 epochs for the CDFS1 and 22 for the CDFS2 (see Table 1 and Fig. 1). We retained only those observations with good seeing, i.e. $F W H M<1.2$ arcsec, in order to optimise the signal-to-noise ratio $(\mathrm{S} / \mathrm{N})$ of the AGN against the host galaxy light, while minimising source blending and position uncertainties. For this reason we excluded the CDFS1 observations made on 2012 Sept. $5(\mathrm{OB}=703670)$ and on $2012 \mathrm{Sept}$ $24(\mathrm{OB}=703698)$ with seeings of 1.28 and 1.44 arcsec, respectively, and the CDFS2 observation made on 2012 Feb. 2 with seeing $1.46 \operatorname{arcsec}(\mathrm{OB}=606780)$.

As we see in Sect. 5, we also exploited a number of overlapping surveys to validate our catalogue of variable 
objects: SWIRE (Lonsdale et al. 2004) and SERVS (Mauduit et al. 2012). For both SWIRE and SERVS, we used the Spitzer Data Fusion catalogues (Vaccari et al. 2010) ${ }^{2}$. These provide a deeper source extraction than the publicly available SWIRE DR5 ${ }^{3}$ and SERVS DR $1^{4}$ catalogues, as well as a wealth of multiwavelength ancillary data. Over the area explored in this paper, data are available in 3.6, 4.5, 5.6, 8, 24, 70, and $160 \mu \mathrm{m}$ bands, and in the $U, g, r, i$, and $z$ filters. We also used the spectral energy distribution (SED) information from the SWIRE photometric redshift catalogue presented in Rowan-Robinson et al. (2013).

Finally, we used the photometric redshift catalogue and SED (in the 0.2 to $10 \mu \mathrm{m}$ observed-frame range) classification published in Hsu et al. (2014) for sources in the ECDFS area. The area explored in Hsu et al. (2014) is the one covered by the Multi-wavelength Survey by Yale-Chile (MUSYC) coverage (Gawiser et al. 2006; Cardamone et al. 2010), which encloses about one-fourth of the CDFS1 area studied in the present work. Inside that area, Hsu et al. (2014) have used the information provided by other campaigns: photometric data from TENIS by Hsieh et al. (2012) and CANDELS by Guo et al. (2013); X-ray data by Xue et al. (2011), Rangel et al. (2013), Lehmer et al. (2005), Virani et al. (2006). More details on the data used in Hsu et al. (2014) are provided in Sect. 5.2. Fig. 1 shows the overlap between the VST CDFS studied in this work and the SWIRE, SERVS, and ECDFS surveys just described.

\section{Catalogue extraction}

For detecting variable sources, we used the method presented in Trevese et al. (2008), which was adapted to work on VST data as described in Paper I. While referring to these papers for details, we summarise the main steps of the procedure here: extraction of the catalogues, aperture correction, masking, and construction of the master catalogue.

For the first step, we used Sextractor to produce source catalogues for each epoch and to measure the photometry in a set of concentric apertures in order to measure the isophotal, aperture, and total source magnitudes. The aperture size for AGN identification should include the majority of the flux from the central source and minimise the contamination from nearby objects or the host galaxy. To build the lightcurve of each source we used the flux measured within a 2 arcsec diameter aperture, centred on the source centroid, which corresponds to about $70 \%$ of the flux from a point-like source given our average seeing. The 2 arcsec diameter is twice the point spread function (PSF) full width at half maximum (FWHM) for the worst images in our dataset (as can be seen in Table 1), allowing to optimise the $\mathrm{S} / \mathrm{N}$ on the aperture flux as shown by, for example, Pritchet \& Kline (1981).

To take the effect of variable seeing into account, we applied aperture corrections to each individual epoch, making use of the growth curves derived from bright stars. The stars were chosen to have circular isophotes, isolated from other sources, and have a low local background. We verified that the PSF is sufficiently uniform across the FOV and that the choice of one specific star does not introduce significant differences. We then corrected all our measurements to $90 \%$ of the total flux ${ }^{5}$.

\footnotetext{
2 http://mattiavaccari.net/df/

3 http://irsa.ipac.caltech.edu/data/SPITZER/SWIRE/

4 http://irsa.ipac. caltech.edu/data/SPITZER/SERVS/

5 This choice is arbitrary and is retained for consistency with Paper I. In fact, the exact value to which we correct is irrelevant for the purpose of variability detection since it is only intended to compensate for seeing
}

The correction technique applied here is based on the assumption that the source is point-like, so it has the effect to over correct extended sources containing faint AGNs because it improperly corrects part of the flux from the galaxy. However, as discussed in Paper I, this effect increases the average variability of the most extended sources by less than $0.01 \mathrm{mag}$ in total rms. The result is that our fixed variability threshold returns a sample somewhat biased towards more extended sources. However, note that the $0.01 \mathrm{mag}$ variability increase is an upper limit, applying only to the most extended sources; in fact, the majority of the variable candidates found in this work are point-like.

The limit magnitude of the shallowest epoch is $r(\mathrm{AB}) \sim 24.5 \mathrm{mag}$ for a source detected at the $5 \sigma$ significance level. The completeness magnitude (of the shallowest epoch) is $r(\mathrm{AB}) \sim 23 \mathrm{mag}$ at the $90 \%$ confidence level, therefore we focus on the variable sources that are more luminous than 23 mag (see Sect. 4). For each epoch, we masked out regions including halos of bright stars, spikes, artefacts, reflection, and residual satellite tracks. A code developed by Huang et al. (2011) was used to produce, for each epoch, specific pixel masks for halos and spikes due to bright stars, taking the star position on the frame and telescope orientation into account. We manually added more pixel masking for extended regions corresponding to other artefacts (i.e. satellites tracks and borders). After the masking procedure, the total number of sources in the master catalogues decreases by about $20 \%$ : we note that in this step we prefer to use a conservative approach to producing a clean sample that favours its purity at the expense of its completeness. We matched the source catalogues of each epoch using a matching radius of 1 arcsec to obtain a single global catalogue to be used for our variability analysis.

Since we aim at building a robust catalogue of variable but persistent sources, we selected only the sources with detections in at least six epochs (out of 27 and 22 epochs in the CDFS1 and CDFS2, respectively). The selection $(r<23 \mathrm{mag}$ and $N_{\text {epochs }} \geq 6$ ) yields 18954 in the CDFS1 and 14340 in the CDFS2. The different number of sources in the two fields is due to the different masked areas and, to a minor extent, to an average lower completeness magnitude (amongst all epochs) in the CDFS2.

\section{Selection of variable sources}

As in Paper I, we determined the average magnitude and the rms of the lightcurves (LC) for each source. We then computed the running average of the individual $\mathrm{rms}\left\langle\sigma_{i}^{\mathrm{LC}}\right\rangle$ and its own standard deviation $\mathrm{rms}_{\left(\sigma_{i}^{\mathrm{LC}}\right)}$ over a 0.5 mag-wide bin. We defined a source as variable if it satisfies the condition: $\sigma_{i}^{\mathrm{LC}}>$ $\left\langle\sigma_{i}^{\mathrm{LC}}\right\rangle+3 \times \operatorname{rms}_{\left(\sigma_{i}^{\mathrm{LC}}\right)}$. Figure 2 shows the running average and the standard deviation as a function of the average source magnitude. The sources above the solid line are flagged as variable candidates. Our approach closely follows the one adopted in other works (Trevese et al. 2008; Bershady et al. 1998). Indeed this method does not allow an a priori statistical estimate of the rate of false positives (as, for instance, in Villforth et al. 2010, using HST data), but this is because we do not know the statistical distribution of our errors (at odds with the much better characterised uncertainties affecting space data, such as those used in Villforth et al. 2010, for instance). While these are dominated by Poissonian uncertainties in the faint regime, at brighter magnitudes the photometric uncertainty is limited by the calibration

effects, thereby normalising all measurements to the same fraction of the total flux. 

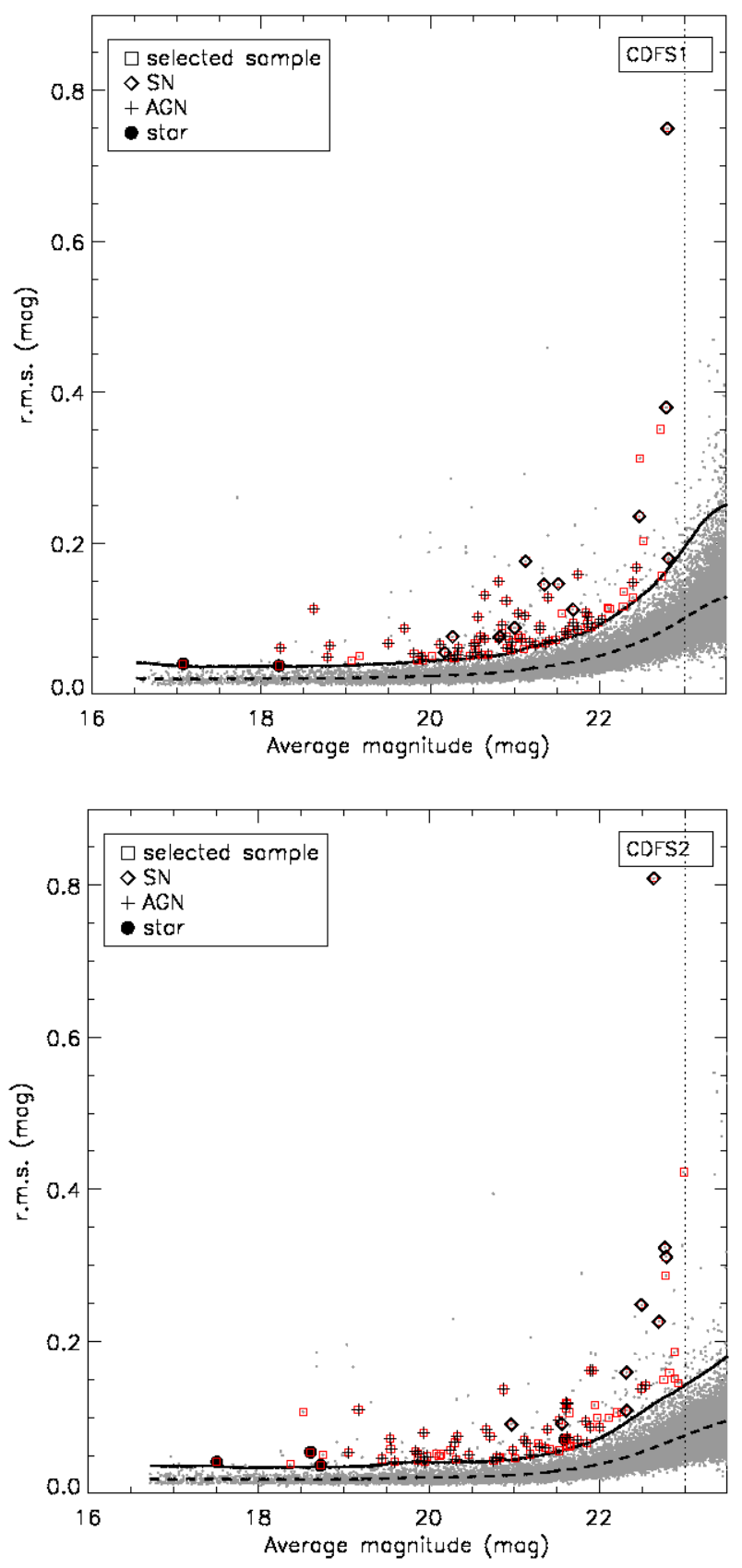

Fig. 2. Root mean square (rms) versus average magnitude of the full masked samples of CDFS1 (top panel) and CDFS2 (bottom panel). Dashed line: running average of the rms; solid line: $3 \sigma$ variability threshold adopted to select variable sources (see text). Sources reported in Table 3 are indicated with the following symbols: squares: all sources of the selected sample; crosses: AGN; circles: stars; diamonds: $\mathrm{SNe}$ (as discussed in Sect. 5).

accuracy of the data (Sect. 2). Thus our approach relies on the definition of a lightcurve rms threshold, and we investigate the nature of the candidates by comparing to optical, IR, X-ray, and spectroscopic surveys (Sect. 5).

To remove sources whose variability may be spurious, we visually inspected each candidate. We retained only those candidates not affected by evident artefacts (e.g. residual stellar diffraction spikes, satellite tracks, strong background gradients, etc.) and/or close neighbours (with distance within 2 arcsec from the source and magnitude lower than $\operatorname{mag}($ source) +1.5 as in De Cicco et al. (2015). For these sources the variability could be due to the contamination of the nearby source and/or centring
Table 2. Number of sources of: (1) selected sample; (2) SN candidates (see Sect. 5.1); (3) Candidates with X-ray detection (Hsu et al. 2014, see Sect. 5.2); (4) AGN candidates (AGN SED in Hsu et al. 2014, see Sect. 5.2); (5) Star candidates (diagnostic $r-i$ versus $3.6 \mu \mathrm{m}$ to $r$ band flux ratios, see Sect. 5.3.1); (6) AGN Lacy et al. (2004) diagnostic using IR colours, see Sect. 5.3.2; (7) AGN candidates confirmed in optical spectroscopy by Boutsia et al. (2009) (BLAGN, Sect. 5.2).

\begin{tabular}{ccc}
\hline \hline & & $N$ \\
\hline$(1)$ & Selected & 175 \\
\hline$(2)$ & $S N$ & $21 / 175$ \\
$(3)$ & X-rays & $12 / 15$ \\
$(4)$ & AGN (SED) & $12 / 15$ \\
$(5)$ & Stars $(r, i, 3.6 \mu \mathrm{m})$ & $6 / 57$ \\
$(6)$ & AGN (IR) & $103 / 115$ \\
$(7)$ & AGN (spectra) & $9 / 9$ \\
\hline
\end{tabular}

issues (for example in very extended sources with irregular profiles for which the identification of the centroid could be problematic). The sample of variable sources is made of 113 candidates in the CDFS1 and 122 candidates in the CDFS2, i.e. 235 candidates in total. To each candidate we attributed a quality flag ranging from 1 to 3 , as in Paper I:

- Flag 1: the candidate is robust, with no evident photometric or aesthetic problems ( $60 \%$ of the 235 candidates);

- Flag 2: the candidate is likely to be variable, and the photometry may be slightly affected by the presence of a nearby companion or by minor aesthetic defects (e.g. faint satellite tracks) (15\%);

- Flag 3: the candidate is very likely spurious, and its variability is uncertain (the remaining $25 \%$ ).

We retained only the candidates with Flags 1 and 2 and obtained a sample of 175 sources (hereafter "selected sample"), including CDFS1 and CDFS2 sources listed in Table 3. We point out that the original criterion for choosing objects with more than six epochs was devised to explore all types of sources included in our survey, including the transients. In principle, lightcurves with different numbers of epochs and $\mathrm{S} / \mathrm{N}$ may have different rms distributions, thus yielding a biased sample. However, we note that $97 \%$ of the sources in our catalogues lack at most three epochs. Moreover, only two sources selected as variable lack more than five epochs (in Table 3, they are the sources with ID: 29449, 290) and both are confirmed SNe from the SUDARE-I SN search (Sect. 5.3).

Figure 3 shows the lightcurves of two sources to illustrate the phenomenology encompassed by this study. The top panel of Fig. 3 displays a candidate whose AGN nature is supported by several diagnostics that will be discussed in Sect. 5 and reported in Table 3, while the bottom panel of Fig. 3 shows a supernova candidate.

\section{Characterisation of variable sources}

In this section, we validate our variable sources by comparison with SN catalogues, X-ray and IR data, and SED information. The main purpose is to assess the purity and the completeness of our sample of 175 candidates and to compare it with the results obtained in Paper I, using additional diagnostics with respect to our previous work.

Table 3 summarises the properties of the selected sample of 175 variable sources. The columns refer to properties, including AGN/non-AGN indicators, discussed in the following sections. 
Table 3. Results on the selected sample.

\begin{tabular}{|c|c|c|c|c|c|c|c|c|c|c|c|c|}
\hline (1) & $\begin{array}{l}\text { RA, Dec } \\
\text { deg } \\
(2)\end{array}$ & $\begin{array}{c}\langle r\rangle \\
\operatorname{mag} \\
(3)\end{array}$ & $\begin{array}{c}\text { Stellarity } \\
\text { (4) }\end{array}$ & $(5)$ & $\begin{array}{l}\text { Quality flag } \\
\text { (6) }\end{array}$ & $\begin{array}{l}S N \\
\mathrm{LC} \\
(7)\end{array}$ & $\begin{array}{l}\text { Star } \\
r, i, 3.6 \mu \mathrm{m} \\
(8)\end{array}$ & $\begin{array}{l}\text { AGN } \\
\text { X-ray } \\
\text { (9) }\end{array}$ & $\begin{array}{l}\text { AGN } \\
\text { spectra } \\
(10)\end{array}$ & $\begin{array}{l}\text { AGN } \\
\text { IR } \\
(11)\end{array}$ & $\begin{array}{l}\text { AGN } \\
\text { SED } \\
(12)\end{array}$ & $\begin{array}{c}\text { Classification } \\
\text { tot } \\
\text { (13) }\end{array}$ \\
\hline 6384 & $03: 27: 55.86-28: 00: 06.5$ & 21.59 & 0.98 & 4.5 & 1 & 0 & 1 & - & - & - & - & -1 \\
\hline 6690 & $03: 28: 30.02-27: 59: 55.7$ & 22.7 & 0.88 & 8.3 & 1 & 1 & 0 & - & - & - & 0 & -2 \\
\hline 13372 & $03: 27: 12.54-27: 55: 09.5$ & 21.67 & 0.03 & 3.3 & 1 & 0 & - & - & - & - & - & - \\
\hline 16666 & $03: 29: 23.05-27: 52: 59.4$ & 22.32 & 0.04 & 7.4 & 1 & 1 & - & - & - & - & - & -2 \\
\hline 20470 & $03: 27: 49.38-27: 50: 15.0$ & 22.64 & 0.96 & 39.0 & 1 & 1 & - & - & - & - & - & -2 \\
\hline 24366 & $03: 27: 53.83-27: 47: 21.5$ & 21.01 & 0.97 & 3.2 & 1 & 0 & - & - & - & - & - & - \\
\hline 27357 & $03: 29: 21.29-27: 45: 09.1$ & 22.26 & 0.84 & 4.2 & 1 & 0 & - & - & - & - & - & - \\
\hline 29449 & $03: 29: 02.74-27: 43: 48.0$ & 22.49 & 0.11 & 11.0 & 1 & 1 & - & - & - & - & - & -2 \\
\hline 30173 & $03: 29: 55.41-27: 43: 18.3$ & 22.82 & 0.97 & 4.4 & 1 & 0 & 0 & - & - & - & 0 & 0 \\
\hline 34660 & $03: 30: 19.49-27: 40: 30.4$ & 21.98 & 0.97 & 5.4 & 1 & 0 & 0 & - & - & - & 0 & 0 \\
\hline 41682 & $03: 29: 38.52-27: 36: 19.0$ & 21.94 & 0.9 & 7.2 & 1 & 0 & 0 & - & - & - & 0 & 0 \\
\hline 44289 & $03: 30: 32.83-27: 34: 46.9$ & 22.99 & 0.89 & 15.8 & 1 & 0 & - & - & - & - & - & - \\
\hline 47325 & $03: 27: 59.79-27: 32: 55.4$ & 21.62 & 0.94 & 3.4 & 1 & 0 & 0 & - & - & - & 0 & 0 \\
\hline 49662 & $03: 30: 26.11-27: 31: 22.2$ & 21.56 & 0.03 & 7.1 & 1 & 1 & - & - & - & - & 0 & -2 \\
\hline 54759 & $03: 30: 27.32-27: 28: 13.2$ & 22.78 & 0.01 & 12.0 & 1 & 1 & - & - & - & - & - & -2 \\
\hline 66439 & 03:31:04.81 -27:20:32.6 & 22.77 & 0.31 & 12.7 & 1 & 1 & - & - & - & - & - & -2 \\
\hline 75327 & $03: 27: 42.78-27: 14: 45.4$ & 22.75 & 0.98 & 4.2 & 1 & 0 & - & - & - & - & - & - \\
\hline 79422 & $03: 28: 49.24-27: 12: 29.4$ & 22.88 & 0.96 & 5.5 & 1 & 0 & - & - & - & - & - & - \\
\hline 2431 & $03: 35: 32.96-28: 03: 24.9$ & 22.12 & 0.98 & 3.9 & 1 & 0 & 0 & - & - & - & - & - \\
\hline 2435 & $03: 35: 15.05-28: 03: 25.5$ & 22.39 & 0.78 & 3.3 & 1 & 0 & 0 & - & - & - & 0 & 0 \\
\hline 5854 & $03: 32: 15.43-28: 01: 23.2$ & 22.47 & 0.03 & 8.4 & 1 & 1 & 0 & 0 & - & - & 0 & -2 \\
\hline 7120 & $03: 34: 49.59-28: 00: 49.7$ & 21.0 & 0.03 & 6.8 & 1 & 1 & 0 & - & - & - & 0 & -2 \\
\hline 15811 & 03:32:08.83-27:55:44.9 & 20.82 & 0.03 & 6.2 & 1 & 1 & 0 & 0 & - & - & 0 & -2 \\
\hline 19508 & $03: 34: 56.87-27: 53: 33.0$ & 21.12 & 0.03 & 15.5 & 1 & 1 & 0 & - & - & - & 0 & -2 \\
\hline 29957 & $03: 33: 57.87-27: 46: 46.8$ & 22.28 & 0.8 & 3.2 & 1 & 0 & - & - & - & - & - & - \\
\hline 60469 & $03: 35: 16.28-27: 29: 49.1$ & 21.05 & 0.03 & 5.1 & 1 & 0 & - & - & - & - & - & - \\
\hline 75131 & $03: 33: 58.45-27: 22: 10.3$ & 21.69 & 0.12 & 6.2 & 1 & 1 & - & - & - & - & - & -2 \\
\hline 75846 & $03: 34: 47.93-27: 21: 42.6$ & 21.51 & 0.04 & 10.0 & 1 & 1 & - & - & - & - & - & -2 \\
\hline 78144 & $03: 34: 38.15-27: 20: 20.6$ & 22.79 & 0.09 & 11.5 & 1 & 1 & - & - & - & - & - & -2 \\
\hline 85754 & $03: 33: 43.87-27: 15: 56.7$ & 22.1 & 0.8 & 4.1 & 1 & 0 & - & - & - & - & - & - \\
\hline 99982 & $03: 33: 26.81-27: 08: 50.5$ & 21.34 & 0.03 & 10.9 & 1 & 1 & - & - & - & - & - & -2 \\
\hline 100845 & 03:33:03.60-27:08:25.4 & 21.55 & 0.93 & 6.1 & 1 & 0 & - & - & - & - & - & - \\
\hline 105093 & 03:35:02.03 -27:06:16.0 & 22.48 & 0.81 & 12.2 & 1 & 0 & - & - & - & - & - & - \\
\hline 2743 & 03:29:59.44-28:02:18.0 & 22.2 & 0.47 & 4.5 & 1 & 0 & - & - & - & - & - & - \\
\hline 28903 & 03:30:07.49 -27:44:09.1 & 22.78 & 0.93 & 10.8 & 1 & 0 & 0 & - & - & - & 0 & 0 \\
\hline 32669 & $03: 30: 43.25-27: 41: 41.3$ & 21.52 & 0.94 & 3.1 & 1 & 0 & 0 & - & - & - & 0 & 0 \\
\hline 36393 & 03:30:12.85 -27:39:31.2 & 22.93 & 0.94 & 3.4 & 1 & 0 & 0 & - & - & - & 0 & 0 \\
\hline 47852 & $03: 27: 19.66-27: 32: 30.4$ & 20.12 & 0.9 & 4.2 & 1 & 0 & - & - & - & - & - & - \\
\hline 67668 & 03:30:06.41 -27:19:48.3 & 22.32 & 0.02 & 4.1 & 1 & 1 & - & - & - & - & - & -2 \\
\hline 69628 & $03: 28: 31.63-27: 18: 34.2$ & 22.11 & 0.03 & 4.6 & 1 & 0 & - & - & - & - & - & - \\
\hline 70019 & $03: 30: 28.14-27: 18: 17.5$ & 22.88 & 0.04 & 3.9 & 1 & 0 & - & - & - & - & - & - \\
\hline 70305 & 03:29:40.46-27:18:06.7 & 20.82 & 0.03 & 3.4 & 1 & 0 & - & - & - & - & - & - \\
\hline 76670 & 03:30:25.09-27:13:57.7 & 21.41 & 0.89 & 3.7 & 1 & 0 & - & - & - & - & - & - \\
\hline 89177 & 03:28:00.28 -27:07:14.1 & 18.52 & 0.87 & 16.6 & 1 & 0 & - & - & - & - & - & - \\
\hline 290 & 03:33:08.76-28:05:04.1 & 22.8 & 0.46 & 25.6 & 1 & 1 & - & - & - & - & - & -2 \\
\hline 525 & 03:35:14.36-28:05:07.4 & 17.08 & 0.96 & 3.6 & 1 & 0 & 1 & - & - & - & - & -1 \\
\hline 618 & $03: 31: 50.76-28: 04: 43.3$ & 22.28 & 0.98 & 4.3 & 1 & 0 & 0 & - & - & - & - & 0 \\
\hline 749 & 03:35:17.41-28:04:39.1 & 18.21 & 0.89 & 3.2 & 1 & 0 & 1 & - & - & - & - & -1 \\
\hline 11324 & $03: 32: 15.84-27: 58: 22.7$ & 22.81 & 0.04 & 3.5 & 1 & 1 & 0 & 0 & - & - & 0 & -2 \\
\hline 72386 & $03: 31: 29.07-27: 23: 36.5$ & 21.1 & 0.88 & 3.0 & 1 & 0 & - & - & - & - & - & - \\
\hline 82256 & $03: 35: 00.42-27: 17: 58.3$ & 21.04 & 0.03 & 5.3 & 1 & 0 & - & - & - & - & - & - \\
\hline 82948 & $03: 33: 39.34-27: 17: 31.3$ & 21.87 & 0.98 & 3.4 & 1 & 0 & - & - & - & - & - & - \\
\hline 84628 & $03: 33: 57.54-27: 16: 32.3$ & 22.73 & 0.97 & 3.0 & 1 & 0 & - & - & - & - & - & - \\
\hline 86007 & $03: 35: 17.02-27: 15: 46.8$ & 22.72 & 0.98 & 11.3 & 1 & 0 & - & - & - & - & - & - \\
\hline 94094 & 03:35:15.03-27:11:44.8 & 22.52 & 0.98 & 6.4 & 1 & 0 & - & - & - & - & - & - \\
\hline
\end{tabular}

Notes. (1) optical ID; (2) coordinates (J2000); (3) average $r$ band magnitude; (4) stellarity index (from Sextractor, see text); (5) significance of variability in rms units; (6) quality flag defined in Sect. 4; (7) SN (identified by their LC and confirmed in SUDARE-I, see Sect. 5.1); (8) stars identified with the diagnostic using $r-i$ versus the $3.6 \mu \mathrm{m}$ to $r$ band flux ratio (see Sect. 5.3.1); (9) X-ray detected/ non-detected sources flagged with 1 and 0, respectively (see Sect. 5.2); (10) AGN validation by optical spectroscopy (Boutsia et al. 2009); (11) sources matching the Lacy et al. (2004) IR criterion for AGN selection (see Sect. 5.3.2); (12) AGN validation by multi-wavelength SED fitting (Rowan-Robinson et al. 2013; Hsu et al. 2014): sources fitted by an AGN template are flagged with 1, otherwise with 0 (slant characters highlight sources in Rowan-Robinson et al. (2013), regular ones the sources in Hsu et al. (2014); (13) Source classification. This flag is obtained summing the individual AGN flags reported in Cols. (9)-(12). It is, thus, a positive number for AGN. The SN are indicated with flag -2 and the stars with flag 1. 
Table 3. continued.

\begin{tabular}{|c|c|c|c|c|c|c|c|c|c|c|c|c|}
\hline (1) & $\begin{array}{l}\text { RA, Dec } \\
\text { deg } \\
(2)\end{array}$ & $\begin{array}{c}\langle r\rangle \\
\text { mag } \\
(3)\end{array}$ & $\begin{array}{c}\text { Stellarity } \\
\text { (4) }\end{array}$ & $\sigma^{*}$ & $\begin{array}{c}\text { Quality flag } \\
\text { (6) }\end{array}$ & $\begin{array}{l}\text { SN } \\
\text { LC } \\
\text { (7) }\end{array}$ & $\begin{array}{l}\text { Star } \\
r, i, 3.6 \mu \mathrm{m} \\
(8)\end{array}$ & $\begin{array}{l}\text { AGN } \\
\text { X-ray } \\
\text { (9) }\end{array}$ & $\begin{array}{l}\text { AGN } \\
\text { spectra } \\
(10)\end{array}$ & $\begin{array}{c}\text { AGN } \\
\text { IR } \\
(11)\end{array}$ & $\begin{array}{l}\text { AGN } \\
\text { SED } \\
(12)\end{array}$ & $\begin{array}{c}\text { Classification } \\
\text { tot } \\
\text { (13) }\end{array}$ \\
\hline 108618 & $03: 34: 10.32-27: 04: 07.0$ & 19.06 & 0.86 & 4.2 & 1 & 0 & - & - & - & - & - & - \\
\hline 110212 & $03: 31: 56.21-27: 02: 56.4$ & 20.02 & 0.9 & 4.0 & 1 & 0 & - & - & - & - & - & - \\
\hline 110606 & $03: 33: 32.72-27: 02: 41.8$ & 20.62 & 0.03 & 3.4 & 1 & 0 & - & - & - & - & - & - \\
\hline 1537 & 03:28:13.01 -28:03:11.5 & 20.84 & 0.92 & 3.6 & 1 & 0 & 0 & - & - & 1 & 1 & 2 \\
\hline 2425 & $03: 30: 17.80-28: 02: 31.3$ & 20.33 & 0.88 & 7.9 & 1 & 0 & 0 & - & - & 1 & 1 & 2 \\
\hline 2535 & $03: 28: 18.32-28: 02: 27.7$ & 20.31 & 0.89 & 6.8 & 1 & 0 & 0 & - & - & 1 & 1 & 2 \\
\hline 2697 & 03:30:15.97 -28:02:19.8 & 19.93 & 0.86 & 8.8 & 1 & 0 & 0 & - & - & 1 & 1 & 2 \\
\hline 6442 & 03:27:11.01 -28:00:02.9 & 20.8 & 0.04 & 3.6 & 1 & 0 & - & - & - & 1 & - & 1 \\
\hline 7134 & 03:30:02.67 -27:59:37.0 & 21.52 & 0.1 & 7.9 & 1 & 0 & 0 & - & - & 1 & 1 & 2 \\
\hline 8056 & 03:30:05.03 -27:58:55.2 & 21.89 & 0.07 & 4.9 & 1 & 0 & 0 & - & - & 1 & 0 & 1 \\
\hline 12153 & $03: 28: 56.48-27: 56: 00.9$ & 21.61 & 0.92 & 9.8 & 1 & 0 & 0 & - & - & 1 & 0 & 1 \\
\hline 16716 & $03: 29: 24.24-27: 52: 56.9$ & 21.62 & 0.92 & 4.7 & 1 & 0 & 0 & - & - & 1 & 0 & 1 \\
\hline 18350 & $03: 29: 40.47-27: 51: 43.6$ & 19.55 & 0.87 & 5.9 & 1 & 0 & 0 & - & - & 1 & 1 & 2 \\
\hline 21592 & $03: 30: 52.20-27: 49: 26.7$ & 21.12 & 0.94 & 6.3 & 1 & 0 & - & - & - & 1 & - & 1 \\
\hline 27638 & $03: 30: 39.70-27: 44: 55.4$ & 21.92 & 0.87 & 11.6 & 1 & 0 & - & - & - & 1 & - & 1 \\
\hline 28499 & $03: 27: 03.63-27: 44: 25.2$ & 19.05 & 1.0 & 6.4 & 1 & 0 & - & - & - & 1 & - & 1 \\
\hline 29899 & $03: 27: 52.98-27: 43: 28.7$ & 21.28 & 0.92 & 5.1 & 1 & 0 & - & - & - & 0 & - & 0 \\
\hline 32210 & $03: 27: 24.94-27: 42: 02.6$ & 19.55 & 0.92 & 8.1 & 1 & 0 & - & - & - & 1 & - & 1 \\
\hline 33242 & $03: 29: 56.70-27: 41: 22.7$ & 20.96 & 0.85 & 9.8 & 1 & 1 & 0 & - & - & 1 & 0 & -2 \\
\hline 34969 & $03: 29: 39.32-27: 40: 20.2$ & 21.74 & 0.92 & 3.9 & 1 & 0 & 0 & - & - & 1 & 0 & 1 \\
\hline 35974 & 03:29:02.09-27:39:46.7 & 21.39 & 0.96 & 7.2 & 1 & 0 & 0 & - & - & 1 & 0 & 1 \\
\hline 39556 & $03: 30: 28.11-27: 37: 36.6$ & 19.87 & 0.87 & 4.6 & 1 & 0 & 0 & - & - & 1 & 1 & 2 \\
\hline 45234 & $03: 28: 37.76-27: 34: 15.4$ & 20.47 & 0.92 & 4.4 & 1 & 0 & 0 & - & - & 1 & 0 & 1 \\
\hline 55181 & $03: 28: 46.21-27: 27: 58.5$ & 21.89 & 0.85 & 12.0 & 1 & 0 & - & - & - & 1 & - & 1 \\
\hline 59923 & $03: 28: 51.64-27: 24: 53.4$ & 22.54 & 0.54 & 4.8 & 1 & 0 & - & - & - & 1 & - & 1 \\
\hline 63050 & $03: 30: 05.75-27: 22: 48.6$ & 21.62 & 0.93 & 9.6 & 1 & 0 & - & - & - & 1 & - & 1 \\
\hline 65830 & $03: 30: 14.36-27: 21: 01.2$ & 20.14 & 0.89 & 4.6 & 1 & 0 & - & - & - & 0 & - & 0 \\
\hline 66781 & 03:30:05.56 -27:20:20.6 & 21.85 & 0.95 & 3.0 & 1 & 0 & - & - & - & 1 & - & 1 \\
\hline 69322 & $03: 27: 36.64-27: 18: 42.7$ & 20.72 & 0.91 & 8.0 & 1 & 0 & - & - & - & 1 & - & 1 \\
\hline 69325 & $03: 28: 38.02-27: 18: 44.0$ & 20.87 & 0.91 & 17.2 & 1 & 0 & - & - & - & 1 & - & 1 \\
\hline 72080 & $03: 27: 21.61-27: 16: 49.3$ & 22.49 & 0.13 & 4.8 & 1 & 0 & - & - & - & 1 & - & 1 \\
\hline 76439 & 03:29:31.00-27:14:07.8 & 21.84 & 0.94 & 6.0 & 1 & 0 & - & - & - & 1 & - & 1 \\
\hline 79350 & $03: 27: 55.68-27: 12: 30.9$ & 22.0 & 0.98 & 4.4 & 1 & 0 & - & - & - & 1 & - & 1 \\
\hline 80235 & $03: 28: 50.23-27: 12: 08.1$ & 19.17 & 0.9 & 16.5 & 1 & 0 & - & - & - & 1 & - & 1 \\
\hline 82885 & $03: 29: 25.35-27: 10: 52.5$ & 19.45 & 0.89 & 4.4 & 1 & 0 & - & - & - & 1 & - & 1 \\
\hline 85284 & $03: 27: 24.97-27: 09: 20.4$ & 20.67 & 0.65 & 9.5 & 1 & 0 & - & - & - & 1 & - & 1 \\
\hline 90638 & $03: 27: 17.13-27: 06: 17.9$ & 19.6 & 0.85 & 3.3 & 1 & 0 & - & - & - & 1 & - & 1 \\
\hline 94735 & 03:30:54.57 -27:03:40.7 & 20.99 & 0.91 & 4.7 & 1 & 0 & - & - & - & 1 & - & 1 \\
\hline 95399 & 03:28:55.70 -27:03:15.2 & 21.6 & 0.95 & 9.0 & 1 & 0 & - & - & - & 1 & - & 1 \\
\hline 2859 & 03:32:31.99-28:03:10.1 & 19.5 & 0.85 & 7.4 & 1 & 0 & 0 & 1 & 1 & 1 & 1 & 4 \\
\hline 4362 & $03: 32: 20.32-28: 02: 15.1$ & 20.56 & 0.91 & 10.7 & 1 & 0 & 0 & 1 & 1 & 1 & 1 & 4 \\
\hline 6814 & $03: 31: 27.79-28: 00: 51.2$ & 21.99 & 0.95 & 3.2 & 1 & 0 & 0 & 1 & 1 & 1 & 1 & 4 \\
\hline 9806 & $03: 34: 39.03-27: 59: 15.4$ & 20.54 & 0.03 & 4.0 & 1 & 0 & - & - & - & 1 & - & 1 \\
\hline 15209 & 03:34:42.03-27:56:05.8 & 20.89 & 0.89 & 6.0 & 1 & 0 & 0 & - & - & 1 & 0 & 1 \\
\hline 17415 & 03:31:45.21 -27:54:35.8 & 20.65 & 0.9 & 14.2 & 1 & 0 & 0 & 1 & - & 1 & 1 & 3 \\
\hline 26084 & 03:33:32.75 -27:49:08.1 & 21.87 & 0.99 & 4.5 & 1 & 0 & - & 1 & 1 & 1 & - & 3 \\
\hline 27645 & 03:35:29.27 -27:48:07.6 & 20.64 & 0.91 & 6.3 & 1 & 0 & - & - & - & 1 & - & 1 \\
\hline 28275 & $03: 32: 59.83-27: 47: 48.4$ & 20.9 & 0.93 & 3.9 & 1 & 0 & - & 1 & 1 & 1 & - & 3 \\
\hline 28432 & $03: 34: 52.50-27: 47: 41.1$ & 21.68 & 0.92 & 4.3 & 1 & 0 & - & - & - & 1 & - & 1 \\
\hline 35698 & $03: 34: 08.26-27: 43: 38.0$ & 20.21 & 0.89 & 3.6 & 1 & 0 & - & - & - & 1 & - & 1 \\
\hline 41079 & $03: 32: 26.49-27: 40: 35.7$ & 19.9 & 0.89 & 4.1 & 1 & 0 & 0 & 1 & 1 & 1 & 1 & 4 \\
\hline 42395 & $03: 34: 42.43-27: 39: 51.6$ & 20.89 & 0.94 & 12.0 & 1 & 0 & 0 & - & - & 1 & 1 & 2 \\
\hline 42982 & 03:32:16.19 -27:39:30.4 & 20.12 & 0.88 & 6.1 & 1 & 0 & 0 & 1 & 1 & 1 & 1 & 4 \\
\hline 46587 & $03: 32: 11.64-27: 37: 26.0$ & 18.78 & 0.86 & 5.1 & 1 & 0 & 0 & 1 & - & 1 & 1 & 3 \\
\hline 47327 & $03: 35: 24.94-27: 36: 55.6$ & 22.02 & 0.91 & 3.4 & 1 & 0 & 0 & - & - & 1 & 0 & 1 \\
\hline 48377 & $03: 35: 28.30-27: 36: 21.5$ & 20.26 & 0.03 & 7.3 & 1 & 1 & 0 & - & - & 0 & 0 & -2 \\
\hline 60497 & $03: 34: 59.84-27: 29: 56.9$ & 19.81 & 0.03 & 4.7 & 1 & 0 & - & - & - & 1 & - & 1 \\
\hline 62569 & $03: 33: 37.75-27: 28: 46.3$ & 20.32 & 0.07 & 3.9 & 1 & 0 & - & - & - & 1 & - & 1 \\
\hline 63435 & $03: 32: 55.70-27: 28: 17.3$ & 22.4 & 0.9 & 4.4 & 1 & 0 & - & - & - & 1 & - & 1 \\
\hline 64886 & $03: 31: 56.25-27: 27: 30.7$ & 21.59 & 0.93 & 3.8 & 1 & 0 & - & - & - & 1 & - & 1 \\
\hline 66489 & $03: 35: 24.18-27: 26: 39.6$ & 22.44 & 0.98 & 5.2 & 1 & 0 & - & - & - & 1 & - & 1 \\
\hline 68199 & $03: 34: 21.38-27: 25: 48.4$ & 21.86 & 0.82 & 3.8 & 1 & 0 & - & - & - & 1 & - & 1 \\
\hline 71431 & 03:33:31.69-27:24:09.0 & 21.74 & 0.88 & 10.0 & 1 & 0 & - & - & - & 1 & - & 1 \\
\hline 79604 & $03: 34: 41.51-27: 19: 29.4$ & 21.38 & 0.96 & 9.0 & 1 & 0 & - & - & - & 1 & - & 1 \\
\hline
\end{tabular}


Table 3. continued.

\begin{tabular}{|c|c|c|c|c|c|c|c|c|c|c|c|c|}
\hline ID & $\begin{array}{l}\text { RA, Dec } \\
\text { deg } \\
\text { (2) }\end{array}$ & $\begin{array}{c}\langle r\rangle \\
\operatorname{mag} \\
(3)\end{array}$ & $\begin{array}{c}\text { Stellarity } \\
\text { (4) }\end{array}$ & $\sigma^{*}$ & $\begin{array}{c}\text { Quality flag } \\
\text { (6) }\end{array}$ & $\begin{array}{l}S N \\
\mathrm{LC} \\
(7)\end{array}$ & $\begin{array}{l}\text { Star } \\
r, i, 3.6 \mu \mathrm{m} \\
(8)\end{array}$ & $\begin{array}{l}\text { AGN } \\
\text { X-ray } \\
\text { (9) }\end{array}$ & $\begin{array}{c}\text { AGN } \\
\text { spectra } \\
(10)\end{array}$ & $\begin{array}{c}\text { AGN } \\
\text { IR } \\
(11)\end{array}$ & $\begin{array}{l}\text { AGN } \\
\text { SED } \\
(12)\end{array}$ & $\begin{array}{c}\text { Classification } \\
\text { tot } \\
\text { (13) }\end{array}$ \\
\hline 79779 & $03: 34: 06.82-27: 19: 23.0$ & 21.83 & 0.93 & 5.1 & 1 & 0 & - & - & - & 1 & - & 1 \\
\hline 81470 & $03: 35: 31.94-27: 18: 25.2$ & 18.81 & 0.95 & 8.0 & 1 & 0 & - & - & - & 1 & - & 1 \\
\hline 83511 & 03:34:13.21 -27:17:11.9 & 21.12 & 0.93 & 7.7 & 1 & 0 & - & - & - & 1 & - & 1 \\
\hline 84399 & $03: 32: 11.40-27: 16: 39.6$ & 21.17 & 0.93 & 3.8 & 1 & 0 & - & - & - & 1 & - & 1 \\
\hline 85224 & $03: 32: 02.44-27: 16: 18.6$ & 18.22 & 0.87 & 7.6 & 1 & 0 & - & - & - & 1 & - & 1 \\
\hline 87751 & 03:33:08.11-27:14:52.4 & 21.29 & 0.09 & 5.6 & 1 & 0 & - & - & - & 1 & - & 1 \\
\hline 90088 & $03: 33: 33.96-27: 13: 46.2$ & 20.68 & 0.91 & 3.3 & 1 & 0 & - & - & - & 1 & - & 1 \\
\hline 90252 & 03:35:09.39-27:13:40.1 & 20.53 & 0.72 & 5.8 & 1 & 0 & - & - & - & 1 & - & 1 \\
\hline 92096 & $03: 34: 27.83-27: 12: 45.2$ & 20.6 & 0.91 & 3.4 & 1 & 0 & - & - & - & 1 & - & 1 \\
\hline 93300 & $03: 35: 02.74-27: 12: 10.3$ & 20.33 & 0.8 & 5.1 & 1 & 0 & - & - & - & 1 & - & 1 \\
\hline 94220 & $03: 31: 39.25-27: 11: 40.3$ & 21.48 & 0.89 & 3.1 & 1 & 0 & - & - & - & 1 & - & 1 \\
\hline 94363 & 03:33:39.51 -27:11:39.0 & 20.81 & 0.91 & 5.8 & 1 & 0 & - & - & - & 1 & - & 1 \\
\hline 94868 & $03: 35: 26.87-27: 11: 22.1$ & 21.75 & 0.94 & 3.7 & 1 & 0 & - & - & - & 1 & - & 1 \\
\hline 95338 & $03: 35: 16.24-27: 11: 08.0$ & 20.59 & 0.9 & 6.9 & 1 & 0 & - & - & - & 1 & - & 1 \\
\hline 96679 & $03: 32: 47.09-27: 10: 36.3$ & 18.62 & 0.81 & 17.0 & 1 & 0 & - & - & - & 1 & - & 1 \\
\hline 99186 & $03: 33: 31.92-27: 09: 16.4$ & 19.7 & 0.93 & 10.4 & 1 & 0 & - & - & - & 1 & - & 1 \\
\hline 99735 & 03:32:03.67-27:08:55.8 & 21.29 & 0.96 & 5.1 & 1 & 0 & - & - & - & 1 & - & 1 \\
\hline 100120 & $03: 35: 29.23-27: 08: 43.4$ & 21.04 & 0.91 & 8.7 & 1 & 0 & - & - & - & 1 & - & 1 \\
\hline 102426 & 03:33:00.69-27:07:39.8 & 20.53 & 0.71 & 5.3 & 1 & 0 & - & - & - & 1 & - & 1 \\
\hline 107237 & $03: 32: 39.74-27: 04: 58.7$ & 20.8 & 0.75 & 15.8 & 1 & 0 & - & - & - & 1 & - & 1 \\
\hline 107399 & $03: 32: 16.66-27: 04: 52.8$ & 21.69 & 0.98 & 4.6 & 1 & 0 & - & - & - & 1 & - & 1 \\
\hline 109802 & $03: 31: 50.59-27: 03: 15.5$ & 19.16 & 0.03 & 5.1 & 1 & 0 & - & - & - & 0 & - & 0 \\
\hline 993 & 03:30:46.15 -28:03:45.6 & 17.51 & 0.97 & 3.8 & 2 & 0 & 1 & - & - & 0 & - & -1 \\
\hline 1158 & $03: 27: 20.05-28: 03: 31.1$ & 18.37 & 0.88 & 3.8 & 2 & 0 & - & - & - & 0 & - & 0 \\
\hline 1806 & $03: 29: 40.20-28: 02: 59.2$ & 19.94 & 0.9 & 4.1 & 2 & 0 & 0 & - & - & 1 & 1 & 2 \\
\hline 3121 & $03: 29: 57.33-28: 02: 07.7$ & 18.61 & 0.89 & 6.8 & 2 & 0 & 1 & - & - & 0 & - & -1 \\
\hline 17120 & $03: 29: 41.57-27: 52: 37.5$ & 21.19 & 0.91 & 3.5 & 2 & 0 & 0 & - & - & 1 & 0 & 1 \\
\hline 22268 & $03: 30: 02.52-27: 48: 58.0$ & 20.25 & 0.95 & 5.6 & 2 & 0 & 0 & - & - & 1 & 1 & 2 \\
\hline 26458 & $03: 28: 51.34-27: 45: 52.2$ & 18.73 & 0.84 & 3.4 & 2 & 0 & 1 & - & - & 0 & 0 & -1 \\
\hline 26548 & $03: 28: 30.68-27: 45: 43.2$ & 18.76 & 0.87 & 6.0 & 2 & 0 & - & - & - & 0 & - & 0 \\
\hline 37366 & $03: 30: 12.05-27: 38: 57.7$ & 20.76 & 0.94 & 3.1 & 2 & 0 & 0 & - & - & 1 & 1 & 2 \\
\hline 39611 & $03: 27: 19.33-27: 37: 34.1$ & 20.31 & 0.89 & 3.4 & 2 & 0 & - & - & - & 1 & - & 1 \\
\hline 51155 & $03: 27: 19.35-27: 30: 24.2$ & 21.37 & 0.92 & 4.2 & 2 & 0 & - & - & - & 1 & - & 1 \\
\hline 64257 & 03:29:41.45 -27:22:05.8 & 20.09 & 0.89 & 4.7 & 2 & 0 & - & - & - & 0 & - & 0 \\
\hline 69697 & 03:30:47.23 -27:18:29.9 & 21.65 & 0.03 & 3.2 & 2 & 0 & - & - & - & 0 & - & 0 \\
\hline 72745 & $03: 30: 41.20-27: 16: 18.7$ & 19.86 & 0.89 & 3.3 & 2 & 0 & - & - & - & 1 & - & 1 \\
\hline 73492 & $03: 28: 47.12-27: 15: 54.1$ & 21.14 & 0.92 & 5.6 & 2 & 0 & - & - & - & 1 & - & 1 \\
\hline 76729 & $03: 28: 42.85-27: 13: 57.3$ & 19.98 & 0.89 & 4.2 & 2 & 0 & - & - & - & 1 & - & 1 \\
\hline 78591 & $03: 30: 51.47-27: 12: 54.8$ & 19.84 & 0.86 & 5.0 & 2 & 0 & - & - & - & 1 & - & 1 \\
\hline 81775 & $03: 28: 45.45-27: 11: 17.3$ & 21.29 & 0.94 & 4.5 & 2 & 0 & - & - & - & 1 & - & 1 \\
\hline 81788 & $03: 27: 23.50-27: 11: 14.0$ & 19.95 & 0.9 & 3.2 & 2 & 0 & - & - & - & 1 & - & 1 \\
\hline 2663 & 03:31:35.44 -28:03:15.9 & 21.24 & 0.9 & 3.1 & 2 & 0 & 0 & 1 & 1 & 1 & 1 & 4 \\
\hline 13037 & $03: 35: 37.95-27: 57: 23.8$ & 21.36 & 0.94 & 3.0 & 2 & 0 & 0 & - & - & 1 & 1 & 2 \\
\hline 19168 & $03: 35: 02.70-27: 53: 33.4$ & 20.28 & 0.03 & 3.2 & 2 & 0 & 0 & - & - & 1 & 0 & 1 \\
\hline 21995 & $03: 31: 35.77-27: 51: 35.0$ & 21.41 & 0.92 & 3.3 & 2 & 0 & 0 & 1 & 1 & 1 & 1 & 4 \\
\hline 48715 & 03:32:59.19-27:36:11.8 & 21.62 & 0.9 & 3.5 & 2 & 0 & - & 1 & - & 1 & 1 & 3 \\
\hline 52751 & $03: 34: 45.88-27: 34: 00.5$ & 21.91 & 0.91 & 3.2 & 2 & 0 & 0 & - & - & 1 & 0 & 1 \\
\hline 76002 & $03: 34: 40.20-27: 21: 38.1$ & 20.84 & 0.04 & 8.0 & 2 & 0 & - & - & - & 1 & - & 1 \\
\hline 76942 & 03:32:19.42-27:21:03.0 & 20.95 & 0.85 & 3.5 & 2 & 0 & - & - & - & 1 & - & 1 \\
\hline 78027 & 03:34:12.02-27:20:29.9 & 19.9 & 0.08 & 3.3 & 2 & 0 & - & - & - & 1 & - & 1 \\
\hline 79292 & $03: 33: 54.77-27: 19: 42.8$ & 21.08 & 0.96 & 4.7 & 2 & 0 & - & - & - & 1 & - & 1 \\
\hline 86211 & $03: 32: 00.12-27: 15: 46.2$ & 19.84 & 0.03 & 3.3 & 2 & 0 & - & - & - & 0 & - & 0 \\
\hline 90077 & $03: 31: 38.46-27: 13: 51.8$ & 20.17 & 0.03 & 4.5 & 2 & 1 & - & - & - & 0 & - & -2 \\
\hline 103927 & 03:32:14.61 -27:06:51.3 & 20.97 & 0.9 & 4.7 & 2 & 0 & - & - & - & 1 & - & 1 \\
\hline 104734 & 03:32:01.24 -27:06:28.5 & 20.47 & 0.03 & 3.5 & 2 & 0 & - & - & - & 1 & - & 1 \\
\hline 24635 & 03:29:03.71 -27:47:08.1 & 21.65 & 0.03 & 8.1 & 2 & 0 & 0 & - & - & - & 0 & 0 \\
\hline
\end{tabular}

\subsection{Contamination by supernovae}

In this section, we consider and quantify the contamination by $\mathrm{SNe}$ in our final sample of 175 variable sources. On the basis of the results derived in Paper I for the COSMOS field, we expect that the variability-selected sample contains a fair fraction of $\mathrm{SNe}(\sim 14 \pm 4 \%)$. The visual (qualitative) inspection of the lightcurves allowed us to identify 24 (out of 175) sources as likely $\mathrm{SNe}$. (They display a rapid luminosity increase, followed by a steep decline and a plateau, bottom panel of Fig. 3.) 

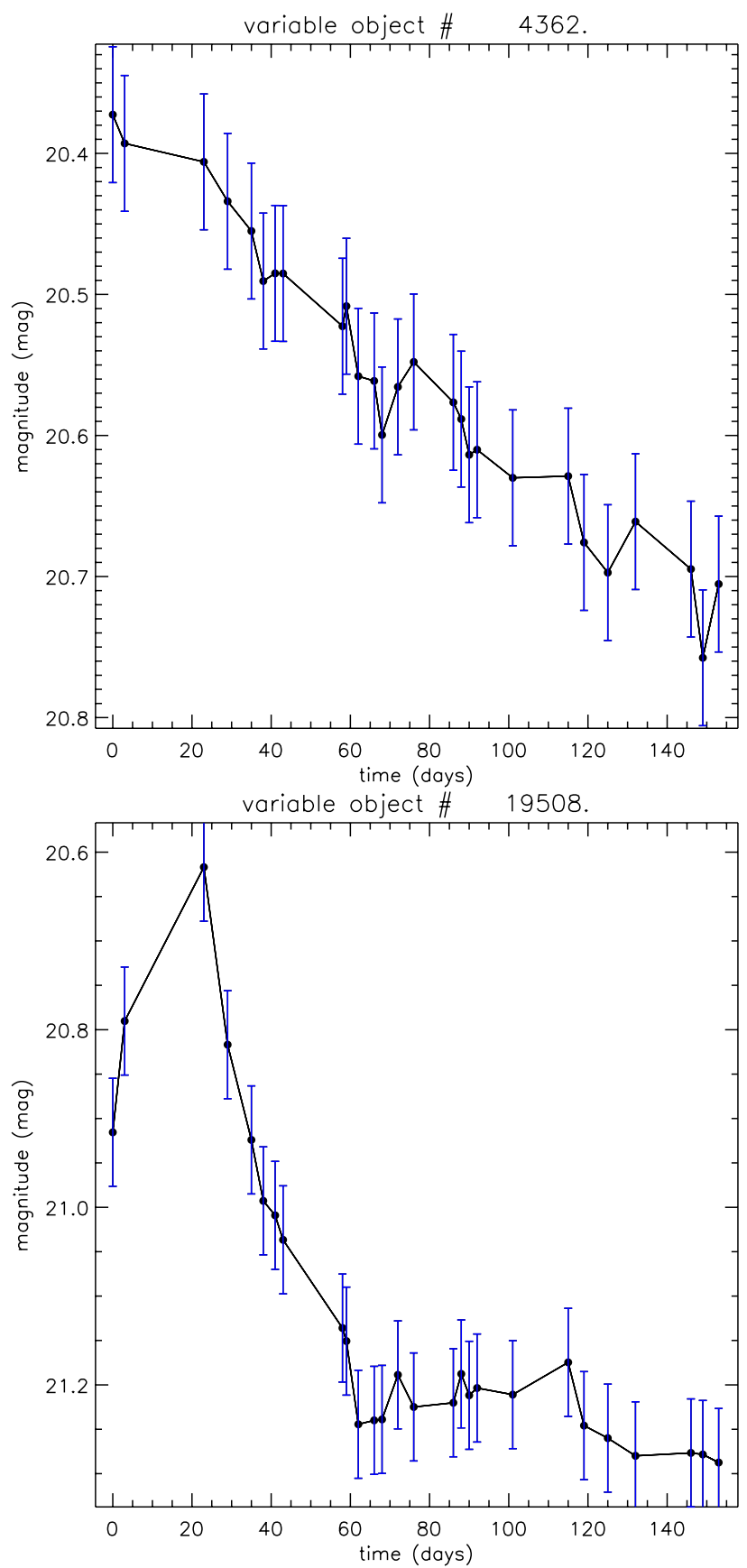

Fig. 3. Top panel: lightcurve of an AGN candidate (ID 4362). Bottom panel: lightcurve of a supernova (ID 19508). The error bars do not represent the formal photometric error except the $\langle\sigma\rangle+3 \times \mathrm{rms}_{\langle\sigma\rangle}$ variability limit and are shown to allow the reader to visualise the significance of the variability.

To validate this identification, we compared our classification with the results of the SN search team (Cappellaro et al., in prep., hereafter SUDARE-I), which adopts a quantitative approach to detecting SNe using the SUDARE-VOICE data. They apply an image subtraction method with respect to a reference epoch, after degrading the images to the lowest resolution image to select variable sources. SUDARE-I selects the sources identified as SNe based on lightcurve fitting in three bands $(g, r, i)$ using a grid of SN templates, where the SN type, the phase, and the reddening are free parameters, and assuming the photometric redshift of the host galaxy as a prior. In this respect the SUDARE-I approach is more general than our visual inspection since it does not rely on the detection of a rising phase, because the free phase parameter and LC fitting allows SNe detected after the peak to be identified. The SN templates used in the fitting are Ia; II, IIn, Ib, and Ic. An estimate of the reddening for the host galaxies is obtained from SED fitting and is applied to the full lightcurve, but we fitted the reddening of the SN independently of the reddening of the host.

The photometric redshift of the host galaxy is derived through SED fitting, using the Eazy code (Brammer et al. 2008), which fits the galaxy SED with a linear combination of synthetic templates. The default template set is optimised to match semianalytic galaxy formation and evolution models that are complete to very faint magnitudes, rather than magnitude-limited spectroscopic samples (see Brammer et al. 2008, for details). The SED fitting is based on a set of $8-12$ broad-band filters ${ }^{6}$. Spectroscopic redshifts are only available for a subsample of galaxies and the collaboration obtained spectra through dedicated observing time for $17 \mathrm{SN}$ candidates. Although the analysis of the SN data is still in progress (and will be presented in Cappellaro et al. in preparation), we find that the measured $\mathrm{SN}$ rate is consistent with published estimates in the literature, within the errors.

We cross-correlated our variable candidates in the selected sample with the list of sources discovered in SUDARE-I. Twenty-one sources, all belonging to our SN candidates, are classified as SN in SUDARE-I as well. There are three additional sources (the ID are 44289, 27638, 105093) with lightcurves similar to those found in SN (as explained before, their lightcurves display a steep increase, followed by a steep decline and a plateau, such as the one shown in Fig. 3, lower panel), but not confirmed as SN in SUDARE-I because the adopted SN templates did not fit their lightcurves well. As we see later, we could not find other multi-band data to use as diagnostics for two of them (105093 and 44289 ). For one source (27 638), one diagnostic instead supports its AGN nature. No additional SN has been found by SUDARE-I within our sample of $r<23$ sources, indicating that our visual inspection was able to identify all bright $\mathrm{SN}$ candidates.

In summary, the fraction of SN listed in the selected sample is $12 \pm 2 \%$ (which corresponds to the $21 / 175 \mathrm{SN}$ found in the selected sample). This fraction agrees, within the error bars $(95 \%$ confidence level), with the results obtained from the COSMOS field analysed in Paper I.

\subsection{SED and X-ray detections}

For characterising the variable sources and the validation of the AGN catalogue, it is necessary to use other diagnostics. Unfortunately, broad SED coverage, optical spectroscopy, or $\mathrm{X}$-ray observations is only available for a small fraction of the survey area: while the ECDFS has been covered by a variety of multi-band and spectroscopic campaigns, the remaining area currently has patchy coverage. We used the information contained in Hsu et al. (2014) to extract X-ray and SED data for our variable candidates located within the ECDFS area, which covers one-eighth of the full region explored in the present paper. The authors computed photometric redshifts via SED fitting for all the galaxies detected in the GOODS/CANDELS area using the deep ( $29 \mathrm{mag}$ ), space-based PSF fitted photometry, presented in Guo et al. (2013). Hsu et al. (2014) also use

\footnotetext{
6 The multi-wavelength coverage depends mainly on the source flux since $u$ and IR data described in Sect. 2 are not as deep as the optical ones.
} 
UV photometry from GALEX and the PSF fitted intermediateband photometry from Subaru, useful for determining the presence of emission lines. For the area outside the CANDELS region, the photometry presented in MUSYC (Gawiser et al. 2006; Cardamone et al. 2010), covering optical, near-IR (NIR), and mid-IR was used, in addition to deeper IR data from the TENIS survey (Hsieh et al. 2012); and GALEX data. For the X-ray band, Hsu et al. (2014) collect information from the 4 Ms source catalogues by Xue et al. (2011) and Rangel et al. (2013), covering the central part of the CDFS area and reaching sensitivity limits of $3.2 \times 10^{-17}, 9.1 \times 10^{-18}$, and $5.5 \times 10^{-17} \mathrm{erg} / \mathrm{cm}^{2} / \mathrm{s}$ in the full $(0.5-8 \mathrm{keV})$, soft $(0.5-2 \mathrm{keV})$, and hard $(2-8 \mathrm{keV})$ bands, respectively. The data for the enlarged ECDFS area are instead collected from the $250 \mathrm{ks}$ catalogues of Lehmer et al. (2005) and Virani et al. (2006), with sensitivity limits of $1.1 \times 10^{-16}$ in the soft band and $3.9 \times 10^{-16} \mathrm{erg} / \mathrm{cm}^{2} / \mathrm{s}$ in the hard band. Photometric redshifts were computed using tuned procedures able to disentangle AGN from stars and galaxies where the X-ray luminosity is produced by stars (see Hsu et al. 2014 for details).

There are only 15 sources in common between the sample presented in Hsu et al. (2014) and our selected sample, using a matching radius of 1 arcsec. The 15 common sources belong to the CDFS1, which encloses the ECDFS. Twelve of the 15 common sources are detected in the X-rays with luminosities and optical/X-ray flux ratios typical of both Types I and II AGNs $\left(-1<\log \left(f_{\text {opt }} / f_{\mathrm{X}}\right)<1\right)$ (see e.g. Mainieri et al. 2002; and Xue et al. 2011) and in agreement with Paper I. Moreover, their SEDs require a strong AGN contribution (in particular in the NIR part of the spectrum, as can be seen in the example shown in the top panel of Fig. 4). All these sources have also been identified as non-SN on the basis of the inspection of their lightcurves in Sect. 5.1. The remaining three sources are undetected in the X-rays (ID: 11324, 5854 and 15811) and their best-fit SED template shows no evidence of a significant AGN contribution. (An SED example is shown in the bottom panel of Fig. 4.) These three sources were identified as $\mathrm{SN}$ according to their lightcurves in Sect. 5.1. Therefore, we conclude that they are SN explosions in normal galaxies. In summary, our variable candidates with a counterpart in the ECDFS region, are either confirmed to contain an AGN according to their broadband properties, or alternatively, when no AGN signature is detected, their lightcurves are consistent with $\mathrm{SN}$.

Two examples of Hsu et al. (2014) fitting are presented for source IDs 2663 and 14321 in our sample (the first one X-ray detected and the second one not). The plots show the photometry, expressed in $\mathrm{AB}$ magnitude and in observed frame, with the bestfitting template. Additionally, nine AGN candidates have optical spectroscopy available from Boutsia et al. (2009) and were identified as broad-line AGN. As we can see from Table 3, the X-ray detections and SED fitting just discussed (Hsu et al. 2014) confirm this identification.

\subsection{IR catalogues (SERVS+SWIRE)}

For further discussion and validation of our catalogue, we used the SERVS (Mauduit et al. 2012) and SWIRE (Lonsdale et al. 2004) samples to exploit the four IRAC bands (the fluxes, respectively, at: $3.6,4.5,5.8,8.0 \mu \mathrm{m}$ ) and the CTIO MOSAIC2 Ugriz optical imaging available through the Spitzer Data Fusion over our VST survey. SERVS and SWIRE overlap an area of $\sim 6 \mathrm{deg}^{2}$ (with 281149 common sources, constituting the catalogue called hereafter SERVS+SWIRE). Almost all the sources in the selected sample fall in the area of SERVS+SWIRE (172 out of 175), and we could find the corresponding IR sources for
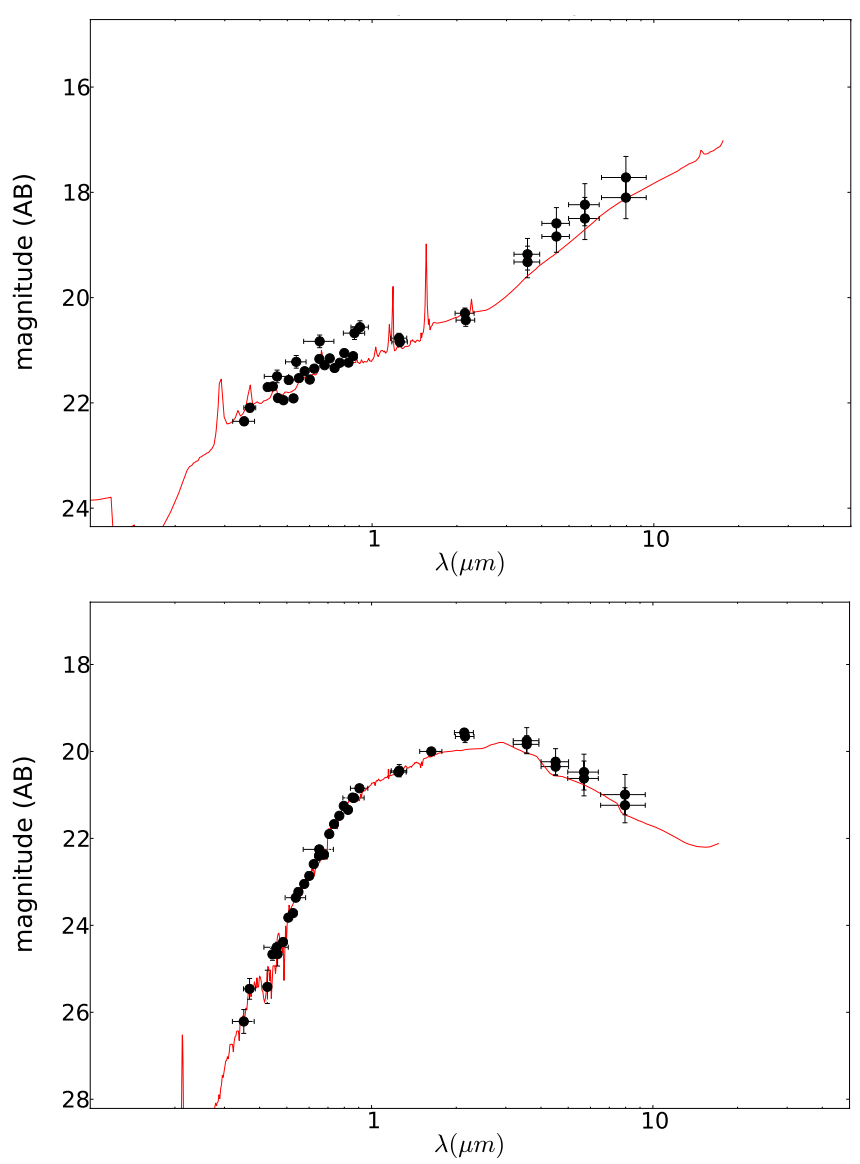

Fig. 4. SED of two sources by Hsu et al. (2014). Top panel: source 2663 (an AGN candidate, see Table 3); bottom panel: source 11324 (a SN candidate).

158 of them, within a radius of 1 arcsec. A 1 arcsec radius is larger than the average offset $(\sim 0.3 \mathrm{arcsec})$ between the optical and IR catalogues but was chosen to ensure that even nearby extended sources with ill-defined centroids (e.g. late-type galaxies) are properly matched. Given the average source density in our fields, we estimate $\sim 1 \%$ false matches. Among the 14 sources in the common area and without SERVS-SWIRE counterparts, there are seven SN (of those discussed in Sect. 5.1): while the transient events (the SN explosions) can be detected using our method, their hosts are likely to be normal galaxies without strong IR emission so not included in the SERVS+SWIRE catalogues.

For the common subset of 158 sources, we used optical and IR colour-colour diagrams to constrain their nature, as described in detail in the following sections.

\subsubsection{Optical-NIR diagnostic}

In Fig. 5 (bottom panel) we compare the $r-i$ colour versus the $3.6 \mu \mathrm{m}$ to $r$ band flux ratio of our variable candidates with the SERVS+SWIRE source catalogue. This diagram has been proposed by Rowan-Robinson et al. (2013) to separate stars from galaxies. The analysis is limited to the 57 objects (of the selected sample) with available data in $r, i$, and $3.6 \mu \mathrm{m}$.

The populations represented in the plot are segregated into two regions: stars and extra-galactic objects. In Fig. 5, a line separates the two regions: below that line, the total number of 

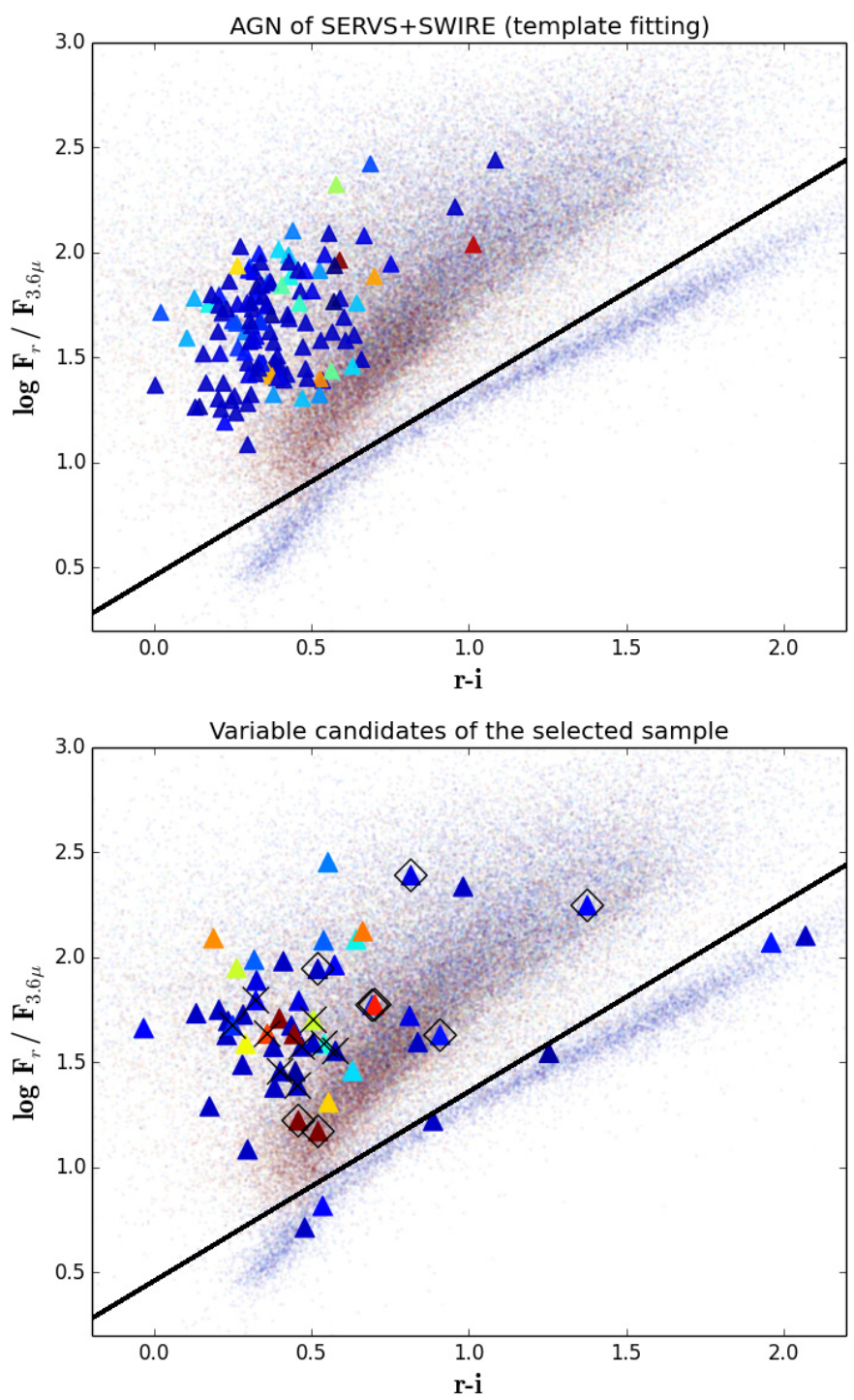

Fig. 5. Flux (F $\lambda$ ) ratios between the $r$ band and $3.6 \mu$ m versus $r-i$ colour. In the top panel, the triangles represent sources flagged as AGN in the SERVS+SWIRE catalog, on the basis of optical/IR template fitting (133 AGN, see Sect. 5.3.1). In the bottom panel the triangles represent the 57 sources belonging to our "selected sample" with available $i, r$, and $3.6 \mu \mathrm{m}$ photometry in the SERVS+SWIRE catalogues (see Sect. 5.3.1). Diamonds and crosses label the SN and X-ray detected sources, respectively (see Sect. 5.2). In both panels, the small points represent the whole SERVS+SWIRE control sample with available $r, i$, $3.6 \mu \mathrm{m}$ photometry (82254 sources). The colours from red (extended sources) to blue (pointlike sources) indicate the increasing stellarity (defined in footnote 9) as measured from the VST images for our "selected sample" or extracted from the SERVS+SWIRE catalogues in the optical $r$ band for the control sample. The solid line divides the plane into the stellar region and the non-stellar region.

stars in SERVS+SWIRE catalogue is 15 times higher than the number of extended objects.

Figure 5 (bottom panel) shows that 6 out of 57 variable sources are along the stellar sequence, so very likely variable stars. We deduce that the fraction of variable stars is probably around $10 \pm 4 \%$ ( 6 out of 57). This result can be compared with that of Sesar et al. (2007) who found $6.4 \%$ of the variable sources identified as RR Lyrae stars, and $18.3 \%$ identified as stars in the stellar sequence. In our sample we can exclude that the six sources in the stellar locus are RR Lyrae because they do not satisfy the selection criterion for their $r-i$ colours: $-0.15<r-i<0.22$ (Sesar et al. 2007). Moreover, the variability timescales of RR Lyrae stars are much shorter than the scales sampled in the present survey (from several hours to one day). The specific nature of our six star candidates is not clear. For one of them (ID 26458), the optical SED classification (reported in Col. 12 of Table 3 ) confirms that there is no AGN contribution. Our $10 \%$ of stars is lower than the $18 \%$ of objects in the stellar sequence found in Sesar et al. (2007). Since Sesar et al. (2007) is based on the SDSS Stripe 82 survey located at lower galactic latitudes, it is expected that more stars were observed in that survey.

Outside the stellar sequence shown in Fig. 5 there are eight SN, confirmed through the lightcurve fitting of SUDARE-I (48377, 19508, 5854, 15811, 7120, 11324, 6690, 33242). There are four extended sources, with erratic lightcurves clearly different from those of SN (from our inspection and Cappellaro et al. in prep). Three of them (those with IDs $7134,8056,19168$ ) are confirmed AGNs according to the IR diagnostic plot discussed in Sect. 5.3.2, while the nature of the last source (ID 24635) is unknown.

Amongst the 51 sources with non-stellar colours, 41 have unresolved profiles according to their stellarity index ${ }^{7}$. Although this diagram is mainly aimed at separating stars from galaxies, it is visually clear that our candidates have colours that differ, on average, from the bulk of the galaxy population. Previous studies (Berta et al. 2006; Tasse et al. 2008) show that both AGNs and high-z galaxies populate the area where our variable sources are located. As a further check, we plot in Fig. 5 (top panel) the AGNs identified in the SERVS+SWIRE catalogue on the basis of the optical/IR template fitting performed by Rowan-Robinson et al. (2013). As can be seen in the bottom panel of the same figure, most of our variable sources not identified as stars have colours that areconsistent with those of AGNs. The non-stellar colors and compactness of our candidates, when coupled with their variability, support the idea that these sources host AGNs, $\mathrm{SNe}$ or some other type of transient source. The lightcurve inspection further allows to remove $\mathrm{SNe}$, thus leaving the AGN classification as the most likely one. This interpretation, however, needs to be validated using additional diagnostics such as those presented in Sects. 5.2 and 5.3.2.

\subsubsection{IR diagnostic}

In this section we make use of the mid-IR colours in order to confirm the identification of our AGN candidates.

Figure 6 shows the diagnostic developed by Lacy et al. (2004, 2007), where the stellarity index is coded from red to blue to represent sources from extended to point-like (as in Fig. 5). Fig. 6 shows sources in the SERVS+SWIRE catalogues and those of the VST-selected sample with available 3.6, 4.5, 5.8, and $8.0 \mu$ photometry (115 sources). Owing to the different dust content and temperature, normal galaxies, star-forming galaxies and AGNs occupy different regions of this diagram. This allows, as shown in Lacy et al. (2004), defining an empirical wedge that encloses a large portion of the AGN population (as mentioned in Sect. 1).

Donley et al. (2012) simulated normal and star-forming galaxies with different levels of AGN contribution to their total emission, in order to derive their IR colours. They find that all the

7 CLASS-STAR given by Sextractor, which is the probability for a source of being point-like -from zero, for extended sources, to one, for point-like sources. 


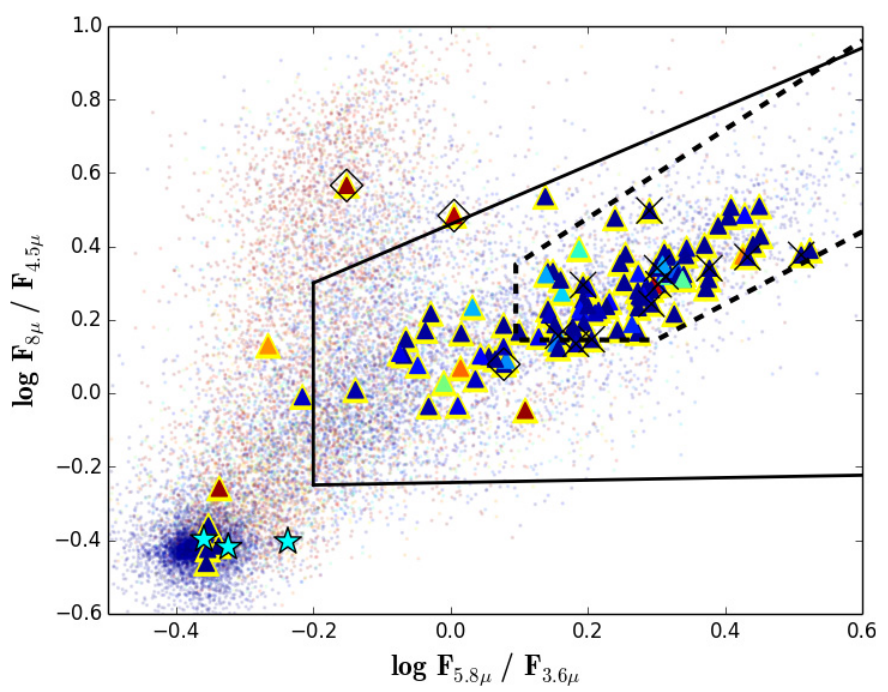

Fig. 6. Flux $\left(\mathrm{F}_{\lambda}\right)$ ratio (logarithmic) at 5.8 and $3.6 \mu$ versus flux ratio at 8 and $4.5 \mu$. Small points: SERVS+SWIRE 18436 sources; triangles (enclosed in yellow edges): 115 sources in common with the selected sample (see Sect. 5.3.2). Cyan stars: stars; diamonds: SN. Crosses: X-ray detected sources. The colours of the triangles and of the small points from red (extended sources) to blue (pointlike sources) indicate the increasing stellarity (as in Fig. 5). The solid line is the Lacy et al. (2004) region and the dashed line the Donley et al. (2012) region.

galaxies with a $>80 \%$ contribution (between 1 and $10 \mu$ ) from an active nucleus are enclosed within the Lacy et al. (2004) wedge. Below this limit some AGNs will be missed by the Lacy et al. (2004) criterion, to the point where only a few sources with an AGN contribution of $<20 \%$ can be found inside the wedge. On the other hand, analysing the correspondence between X-ray and IR selection, they found that the Lacy region also contains a significant fraction of non-X-ray detected sources, some of which are likely starbursts. This result is also confirmed by Yun et al. (2008), who found that submillimetre galaxies (which are dominated by starbursts) contaminate the Lacy et al. (2004) region. To improve the purity of the IR-selected AGN sample, Donley et al. (2012) defined a more restrictive criterion for reducing the starburst contamination to IR-selected AGN samples, which is shown in Fig. 6.

Out of the 115 sources of the selected sample represented in the plot, 103 lie within the Lacy et al. (2004) wedge, marking them as likely AGN. Although starburst galaxies contaminate this region, their variability supports the AGN classification.

However, false-positive variability detections are still possible. Since the Lacy wedge is contaminated by starbursts, we expect that some of the false positive objects may not be AGN even if they lie in this region. To estimate the number of such spurious confirmations, we need to evaluate the number of falsepositive variable sources that are at the same time contaminants lying within the Lacy wedge. The contamination rate of the Lacy wedge is strongly dependent on the flux limits of the survey, as shown in Donley et al. (2012). In surveys with fluxes of $>50 \mu \mathrm{Jy}$ at $5.8 \mu \mathrm{m}$, the contamination in the majority of the Lacy area is of the order of $8 \%$ (see Lacy et al. 2007). Decreasing the flux limits down to $\sim 11 \mu \mathrm{Jy}$, the contamination increases especially at bluer colors (i.e. towards the bottom left edge of the wedge, see Fig. 8 of Donley et al. 2012). The $5.6 \mu \mathrm{m}$ flux limit of our master catalogue (with $r<23 \mathrm{mag}$ ) is $\sim 10 \mu \mathrm{Jy}$, so we expect potential contamination up to $90 \%$ at the leftmost edge of the
Lacy region but less than $10 \%{ }^{8}$ within the Donley wedge. Since our variable sources tend to lie far from the Lacy edges (as can be seen in Fig. 6), we can assume for our estimates an average contamination of $50 \%$.

The percentage of spurious variable sources within the Lacy wedge can be estimated assuming the worst case scenario in which all our sources above the variability threshold are false positives (1\% of parent sample). Since the number of sources within the Lacy wedge is $\sim 2000$, we estimate at most 20 false variable objects. Of these only $50 \%$ are expected to be also contaminants with colours consistent with AGNs (as discussed above), so we estimate less than ten spurious sources and an upper limit of $10 \%(10 / 103)$ on the fraction of erroneously confirmed AGN.

We also note from Fig. 6 that the average stellarity index of the variable candidates inside the Lacy et al. (2004) region decreases towards the left-hand side of the diagram; i.e., the sources become more extended, indicating that they are likely to be low redshift galaxies where the nucleus does not dominate the overall emission. According to Trevese et al. (2008), many "variable galaxies", i.e. extended variable sources, are narrow emission line galaxies with a low ionisation narrow emission region (LINER). The majority of point-like sources lie within the Donley et al. (2012) wedge, strengthening the view that the Donley et al. (2012) region is occupied prevalently by AGNdominated galaxies. Indeed the Donley criterion allows rather complete ( $\sim 88 \%$ with respect to X-ray selection) samples of luminous AGNs to be identified but misses low-luminosity AGNs with host-dominated SEDs (see Donley et al. 2012, for details). Moreover, all the X-ray detected sources (discussed in Sect. 5.2) are found inside the Donley wedge, further supporting the view that their X-ray emission is not due to star formation but to the active nucleus. These results suggest a strong correspondence between variability, X-ray, and IR selection criterion, at least for AGN-dominated galaxies.

Out of the 12 sources located outside the AGN area of Lacy et al. (2004), three are point-like sources classified as stars in the plot $r-3.6$ versus $r-i$ discussed in Sect. 5.3.1 (ID: 26458, 3121, and 993), and two (IDs 90077 and 48377) have been identified as $\mathrm{SN}$ in Sect. 5.1, and are thus likely star-forming galaxies hosting SNe. The remaining seven sources are difficult to classify with this diagnostic: four pointlike sources (IDs 65830, 1158, 26548, 64257 in Table 3) are located in the "blue clump" in the lower left of the diagram; because of their non-AGN colours and pointlike profiles they can be unresolved galaxies or stars, see Donley et al. (2012). The remaining three outliers are extended sources: source 109802 (very extended source, with strong variability significance, see Table 3), source 86211 (it has a nearby companion and for this reason it has been flagged with quality flag 2), and source 69697. (This source has quality flag 2 and displays only marginal variability.) The location of these three extended sources in the diagnostic plot is similar to that of simulated sources with redshift between $\sim 1$ and $\sim 2$ and AGN contribution below 40\% (Donley et al. 2012).

Summarising, according to the IR diagnostic discussed in this section, our sample contains 103/115 likely AGN, with at most ten contaminants.

$815 \%$ of the X-ray undetected sources - which in turn are $50 \%$ of the total sample - have colors consistent with star formation as discussed in Fig. 8 and Sect. 9.1 of Donley et al. (2012). 


\section{Summary and conclusions}

In this paper we used the VST SUDARE-VOICE multi-epoch observations in the CDFS area of $2 \mathrm{deg}^{2}$ to select a sample of AGN via their optical variability. Variability is a valuable tool for selecting AGN candidates, as shown in Sarajedini et al. (2003, 2006, 2011), for example.

Trevese et al. (2008) used ground-based observations over nine epochs of the ESO/MPI telescope in the framework of the STRESS survey. The variability study conducted in that context in the V band produced results consistent with those obtained in independent X-ray and IR variability-selection methods. They find that the variability selection produces a sample with $44 \%$ completeness (with respect to the X-ray selected sample with $L_{\mathrm{X}}>10^{42} \mathrm{erg} / \mathrm{s}$ and known spectra, see Trevese et al. 2008 for details) and less than $40 \%$ contamination. The temporal sampling and the number of epochs used in that work were not high enough to limit the contamination and assure a higher level of completeness. By increasing the number of epochs, the temporal sampling, and the photometric accuracy, variability surveys can reach far higher completeness: for example, $\sim 90 \%$ completeness was reached in Sesar et al. (2007) -with respect to the sample of spectroscopically confirmed SDSS quasars from Stripe 82- exploiting observations spanning a period of six years (one or two observations per season).

In this paper, we have presented a new sample of AGN selected through variability and we have assessed its purity on the basis of IR colors, optical spectroscopic and X-ray surveys over the explored region. The data used here are the VST observations of the CDFS region acquired by the SUDARE-VOICE collaboration. We measured the lightcurves of all sources over the four-to-five- month baseline covered by the VST observations. Our selection method relied on the comparison of the rms variability of each source to the average of the entire population of sources with comparable magnitude, selecting as variable those sources with an rms larger than three standard deviations from the average rms.

From the catalogues of sources detected in more than six epochs and $r<23 \mathrm{mag}$, we identified 175 candidates (we refer to them as "selected sample"). To validate the sample, we used information available both within the VST-SUDARE consortium and in the literature (Hsu et al. 2014; Boutsia et al. 2009; Lonsdale et al. 2004; Mauduit et al. 2012).

We found that:

1. $12 \pm 2 \%(21 / 175)$ of the selected sample are classified as $\mathrm{SN}$, based on both visual inspection of the lightcurves and template fitting by the SUDARE-I collaboration (Cappellaro et al., in prep.).

2. An additional $3 \%(6 / 175)$ of the selected sample includes stars, based on the optical-IR diagnostic $(r-i$ versus the $3.6 \mu \mathrm{m}$ to $r$ band flux ratio) of Rowan-Robinson et al. (2013). This diagram also shows that most of the remaining variable sources have colours consistent with AGN.

3. For the 115 objects with detections in all four IRAC bands, 103 (59\% of the selected sample) have colours that are consistent with AGN emission. Although the Lacy et al. (2004) criterion allows for contamination by starforming galaxies, the detection of variability supports the idea that in these sources a significant fraction of the IR emission is produced by an AGN. We estimate an upper limit of $10 \%$ to the number of erroneously confirmed AGNs. We found that the most compact sources tend to have colours consistent with the more restrictive criterion proposed by Donley et al. (2012), which are AGN-dominated objects, while the most extended sources lie on the IR diagram where some contamination from starburst/host galaxy light is expected. Outside the wedge of Lacy et al. (2004) we identified twelve sources: five of these are among the $\mathrm{SNe}$ and stars discussed above, and the remaining seven are objects without clear AGN/star/SN signatures (except possibly for the variability properties) and may be spurious detections or normal galaxies hosting very low luminosity AGN.

4. In the selected sample there are 15 sources within the ECDFS area for which we have SED, X-ray, and spectroscopic information presented in Hsu et al. (2014) and Boutsia et al. (2009). In particular we found:

- twelve sources with a clear AGN signature in their SED, which are also X-ray detected. All of them are AGN, based on the IR selection (see Table 3);

- three variable sources with purely galactic (non-AGN) SED and not detected in the X-ray band; this is consistent with our previous SN classification based on lightcurves;

- nine variable sources confirmed by the optical spectroscopy presented in Boutsia et al. (2009), which have been classified as BLAGN. All of them are already classified as AGN based on the IR selection, X-ray selection, and SED properties. This result is consistent with the one shown in Paper I: the majority of the variable sources in COSMOS are BLAGN (as expected given the selection method).

Overall, the total number of candidates for which we could employ the diagnostics discussed in Sect. 5 (as can be seen in Table 3) is 137 out of a total 175 selected candidates. Of the 137 sources, we found 102 AGN confirmed by at least one diagnostic, which is $58 \%$ of the selected sample. Among the remaining sources six are stars, and nine are SN. Column 13 of Table 3 indicates the sum of the AGN flags in Cols. 9-12. For AGN, this is a positive number that counts the number of positive diagnostics. The flag is instead negative for stars and SN. The percentage of confirmed AGN, as well as those of identified contaminants are consistent with the results presented in Paper I for the COSMOS region. We also found 18 objects whose AGN nature could not be verified by the diagnostics investigated in this work. There is, finally, a group of 38 sources (among which $12 \mathrm{SN}$ ) for which we could not employ any diagnostic other than our lightcurve inspection.

Our results are useful in predicting the expected performance of future monitoring surveys similar in depth and cadence to our own, to assemble samples of AGNs based on variability alone. Twelve percent of the selected sample is composed of $\mathrm{SNe}$ (identified through the inspection of the lightcurves), which were removed from the sample. In our dataset, $4 \%(6 / 154)$ of the non-SN sources are stars, while 66\% (102/154) are confirmed AGNs based on the available diagnostics. We can thus estimate an upper limit to the contamination of the variabilityselected AGN sample of $\simeq 34 \%$, assuming a worst-case scenario where all unconfirmed variable sources (excluding SN) are nonAGNs. However we point out that restricting the analysis to the sources with available multi-wavelength ancillary information, the purity of our sample is $\sim 80 \%$ (102 confirmed AGNs/128 non-SN). While some of the IR confirmed AGNs can be spurious (as discussed in Sect. 5.3.2) we estimate that this effect would yield a false positive rate of at most $10 \%$. Our results agree with those found in Paper I on the COSMOS field where almost all sources could be identified by means of multi-wavelength information and/or spectroscopy; in that case, we showed that our variability-selected sample reached a final purity of $93 \%$ 
(Paper I). A spectroscopic follow-up of the unconfirmed sources is desirable to conclusively verify this result for the CDFS area.

The completeness of the variability-selected survey presented in this work is $22 \%$, computed with respect to the IR selection of Donley et al. (2012) described in Sect. 5.3.2. In Paper I, the completeness (computed with respect to X-ray samples) has been estimated to be $15 \%$ for a five-month baseline; the two results are thus in broad agreement considering that the completeness is estimated with respect to different reference populations (for instance, only $50 \%$ of the IR selected sources by Donley are detected in X-rays, but at the same time the IR criterion tends to favour luminous AGNs with $L_{\mathrm{X}}>10^{43} \mathrm{erg} \mathrm{s}^{-1}$ ). The completeness found in the present work and in Paper I represents a lower limit to the potential completeness of the variability-selected survey that improves extending the monitoring baseline, as we have seen in previous papers (e.g. Sesar et al. 2007 and Paper I). The new observations that are currently being acquired for the COSMOS field (P.I.: G. Pignata) with VST will allow us to directly compare these results using a three-year long monitoring baseline.

Summarising, this paper makes use of the VST observations, together with the IR photometry, SED fitting and X-ray information where available to confirm the nature of the AGN candidates. The IR data, available over the full survey area, allow confirming the consistency of the variability selection with the IR colour selection method, while the detection of variability may prove useful to detect the presence of an AGN in IR-selected starburst galaxies.

By applying the variability-selection method used here to future datasets such as LSST, it will be possible to complete the AGN demography including strongly variable sources and also AGN, which could be confused with stars of similar colours.

Acknowledgements. We acknowledge the Department of Physics of the University Federico II Naples, Italy. G.L. and E.C. acknowledge the financial support by PRIN 2011 "Cosmology with Euclid Mission". We acknowledge PRIN INAF 2011 "Galaxy evolution with VST"; we also acknowledge the support from PRIN 2014 (P.I. N. Napolitano). This work was supported by the European Commission Research Executive Agency FP7-SPACE-2013-1 Scheme (Grant Agreement 607254 - Herschel Extragalactic Legacy Project - HELP) and by the Italian Ministry for Foreign Affairs and International Cooperation Joint Research Projects of Particular Relevance (Grant Agreement ZA14GR02 - "Mapping the Universe on the Pathway to SKA"). The authors acknowledge L. Greggio for support at OmegaCAM. Funding for SDSS-III has been provided by the Alfred P. Sloan Foundation, the Participating Institutions, the National Science Foundation, and the U.S. Department of Energy Office of Science. The SDSS-III web site is http://www.sdss3.org/. SDSS-III is managed by the Astrophysical Research Consortium for the Participating Institutions of the SDSS-III Collaboration including the University of Arizona, the Brazilian Participation Group, Brookhaven National Laboratory, Carnegie Mellon University, University of Florida, the French Participation Group, the German Participation Group, Harvard University, the Instituto de Astrofisica de Canarias, the Michigan State/Notre Dame/JINA Participation Group, Johns Hopkins University, Lawrence Berkeley National Laboratory, Max Planck Institute for Astrophysics, Max Planck Institute for Extraterrestrial Physics, New Mexico State University, New York University, Ohio State University, Pennsylvania State University, University of Portsmouth, Princeton University, the Spanish Participation Group, University of Tokyo, University of Utah, Vanderbilt University, University of Virginia, University of Washington, and Yale University. G.P. acknowledges support provided by the Millennium Institute of Astrophysics (MAS) through grant IC120009 of the Programa Iniciativa Cientifica Milenio del Ministerio de Economia, Fomento y Turismo de Chile. We thank the anonymous referee for constructive comments that were helpful for improving the paper.

\section{References}

Ajello, M., Alexander, D. M., Greiner, J., et al. 2012, ApJ, 749, 21 Antonucci, M., Talavera, A., Vagnetti, F., et al. 2015, A\&A, 574, A49
Bershady, M. A., Trevese, D., \& Kron, R. G. 1998, ApJ, 496, 103 Berta, S., Rubele, S., Franceschini, A., et al. 2006, A\&A, 451, 881 Botticella, M. T., Cappellaro, E., Pignata, G., et al. 2013, The Messenger, 151, 29

Boutsia, K., Leibundgut, B., Trevese, D., \& Vagnetti, F. 2009, A\&A, 497, 81 Brammer, G. B., van Dokkum, P. G., \& Coppi, P. 2008, ApJ, 686, 1503 Cardamone, C. N., van Dokkum, P. G., Urry, C. M., et al. 2010, ApJS, 189, 270 Cerviño, M., Mas-Hesse, J. M., \& Kunth, D. 2002, A\&A, 392, 19

Comastri, A., Ranalli, P., Iwasawa, K., et al. 2011, A\&A, 526, L9

De Cicco, D., Paolillo, M., Covone, G., et al. 2015, A\&A, 574, A112

Donley, J. L., Koekemoer, A. M., Brusa, M., et al. 2012, ApJ, 748, 142

Eisenstein, D. J., Weinberg, D. H., Agol, E., et al. 2011, AJ, 142, 72

García-González, J., Alonso-Herrero, A., Pérez-González, P. G., et al. 2014, ArXiv e-prints [arXiv: 1411.7556]

Gawiser, E., van Dokkum, P. G., Herrera, D., et al. 2006, ApJS, 162, 1

Geha, M., Alcock, C., Allsman, R. A., et al. 2003, AJ, 125, 1

Giacconi, R., Zirm, A., Wang, J., et al. 2002, ApJS, 139, 369

Gopal-Krishna \& Subramanian, K. 1991, Nature, 349, 766

Grado, A., Capaccioli, M., Limatola, L., \& Getman, F. 2012, Mem. Soc. Astron. Ital. Suppl., 19, 362

Graham, M. J., Djorgovski, S. G., Drake, A. J., et al. 2014, MNRAS, 439, 703

Guo, Y., Ferguson, H. C., Giavalisco, M., et al. 2013, ApJS, 207, 24

Hawkins, M. R. S. 1983, MNRAS, 202, 571

Hsieh, B.-C., Wang, W.-H., Hsieh, C.-C., et al. 2012, ApJS, 203, 23

Hsu, L.-T., Salvato, M., Nandra, K., et al. 2014, ApJ, 796, 60

Huang, Z., Radovich, M., Grado, A., et al. 2011, A\&A, 529, A93

Ivezić, Ž., Lupton, R. H., Anderson, S., et al. 2003, Mem. Soc. Astron. It., 74 978

Jiménez-Bailón, E., Santos-Lleó, M., Dahlem, M., et al. 2005, A\&A, 442, 861

Kawaguchi, T., Mineshige, S., Umemura, M., \& Turner, E. L. 1998, ApJ, 504, 671

Kuijken, K. 2011, The Messenger, 146, 8

Lacy, M., Storrie-Lombardi, L. J., Sajina, A., et al. 2004, ApJS, 154, 166

Lacy, M., Petric, A. O., Sajina, A., et al. 2007, AJ, 133, 186

Lehmer, B. D., Brandt, W. N., Alexander, D. M., et al. 2005, ApJS, 161, 21

Lehmer, B. D., Xue, Y. Q., Brandt, W. N., et al. 2012, ApJ, 752, 46

Lonsdale, C., Polletta, M. D. C., Surace, J., et al. 2004, ApJS, 154, 54

Luo, B., Bauer, F. E., Brandt, W. N., et al. 2008, ApJS, 179, 19

Mainieri, V., Bergeron, J., Hasinger, G., et al. 2002, A\&A, 393, 425

Markarian, B. E. 1967, Astrofizika, 3, 55

Mauduit, J.-C., Lacy, M., Farrah, D., et al. 2012, PASP, 124, 1135

Paolillo, M., Schreier, E. J., Giacconi, R., Koekemoer, A. M., \& Grogin, N. A. 2004, ApJ, 611, 93

Perez-Olea, D. E., \& Colina, L. 1996, ApJ, 468, 191

Peterson, B. M. 2001, in Advanced Lectures on the Starburst-AGN, eds. I. Aretxaga, D. Kunth, \& R. Mújica, 3

Polletta, M. D. C., Wilkes, B. J., Siana, B., et al. 2006, ApJ, 642, 673

Pritchet, C., \& Kline, M. I. 1981, AJ, 86, 1859

Qian, S. J., Quirrenbach, A., Witzel, A., et al. 1991, A\&A, 241, 15

Rangel, C., Nandra, K., Laird, E. S., \& Orange, P. 2013, MNRAS, 428, 3089

Rowan-Robinson, M., Gonzalez-Solares, E., Vaccari, M., \& Marchetti, L. 2013, MNRAS, 428, 1958

Sanders, D. B., Phinney, E. S., Neugebauer, G., Soifer, B. T., \& Matthews, K. 1989, ApJ, 347, 29

Sarajedini, V. L., Gilliland, R. L., \& Kasm, C. 2003, ApJ, 599, 173

Sarajedini, V. L., Koo, D. C., Phillips, A. C., et al. 2006, ApJS, 166, 69

Sarajedini, V. L., Koo, D. C., Klesman, A. J., et al. 2011, ApJ, 731, 97

Sesar, B., Ivezić, Ž., Lupton, R. H., et al. 2007, AJ, 134, 2236

Shakura, N. I., \& Sunyaev, R. A. 1976, MNRAS, 175, 613

Stern, D., Eisenhardt, P., Gorjian, V., et al. 2005, ApJ, 631, 163

Tasse, C., Le Borgne, D., Röttgering, H., et al. 2008, A\&A, 490, 879

Trevese, D., Pittella, G., Kron, R. G., Koo, D. C., \& Bershady, M. 1989, AJ, 98, 108

Trevese, D., Kron, R. G., Majewski, S. R., Bershady, M. A., \& Koo, D. C. 1994, ApJ, 433, 494

Trevese, D., Boutsia, K., Vagnetti, F., Cappellaro, E., \& Puccetti, S. 2008, A\&A, 488, 73

Ulrich, M.-H., Maraschi, L., \& Urry, C. M. 1997, ARA\&A, 35, 445

Vaccari, M., Marchetti, L., Franceschini, A., et al. 2010, A\&A, 518, L20

Veilleux, S., \& Osterbrock, D. E. 1987, ApJS, 63, 295

Veron, P., \& Hawkins, M. R. S. 1995, A\&A, 296, 665

Villforth, C., Koekemoer, A. M., \& Grogin, N. A. 2010, ApJ, 723, 737

Villforth, C., Sarajedini, V., \& Koekemoer, A. 2012, MNRAS, 426, 360

Virani, S. N., Treister, E., Urry, C. M., \& Gawiser, E. 2006, AJ, 131, 2373

Xue, Y. Q., Luo, B., Brandt, W. N., et al. 2011, ApJS, 195, 10

Young, M., Brandt, W. N., Xue, Y. Q., et al. 2012, ApJ, 748, 124

Yun, M. S., Aretxaga, I., Ashby, M. L. N., et al. 2008, MNRAS, 389, 333 